\title{
Neural and Synaptic Defects in slytherin, a Zebrafish Model for Human Congenital Disorders of Glycosylation
}

\author{
Yuanquan Song ${ }^{1}$, Jason R. Willer ${ }^{3}$, Paul C. Scherer ${ }^{1}$, Jessica A. Panzer ${ }^{1}$, Amy Kugath ${ }^{1}$, Emmanuel \\ Skordalakes $^{2}$, Ronald G. Gregg ${ }^{3}$, Gregory B. Willer ${ }^{3}$, Rita J. Balice-Gordon ${ }^{1 *}$
}

1 Department of Neuroscience, University of Pennsylvania School of Medicine, Philadelphia, Pennsylvania, United States of America, 2 Wistar Institute, Philadelphia, Pennsylvania, United States of America, 3 Department of Biochemistry and Molecular Biology, University of Louisville, Louisville, Kentucky, United States of America

\begin{abstract}
Congenital disorder of glycosylation type IIC (CDG IIc) is characterized by mental retardation, slowed growth and severe immunodeficiency, attributed to the lack of fucosylated glycoproteins. While impaired Notch signaling has been implicated in some aspects of CDG Ilc pathogenesis, the molecular and cellular mechanisms remain poorly understood. We have identified a zebrafish mutant slytherin (srn), which harbors a missense point mutation in GDP-mannose 4,6 dehydratase (GMDS), the rate-limiting enzyme in protein fucosylation, including that of Notch. Here we report that some of the mechanisms underlying the neural phenotypes in srn and in CGD Ilc are Notch-dependent, while others are Notchindependent. We show, for the first time in a vertebrate in vivo, that defects in protein fucosylation leads to defects in neuronal differentiation, maintenance, axon branching, and synapse formation. Srn is thus a useful and important vertebrate model for human CDG Ilc that has provided new insights into the neural phenotypes that are hallmarks of the human disorder and has also highlighted the role of protein fucosylation in neural development.
\end{abstract}

Citation: Song Y, Willer JR, Scherer PC, Panzer JA, Kugath A, et al. (2010) Neural and Synaptic Defects in slytherin, a Zebrafish Model for Human Congenital Disorders of Glycosylation. PLoS ONE 5(10): e13743. doi:10.1371/journal.pone.0013743

Editor: Edward Giniger, National Institutes of Health (NIH), United States of America

Received May 21, 2010; Accepted August 22, 2010; Published October 29, 2010

Copyright: (c) 2010 Song et al. This is an open-access article distributed under the terms of the Creative Commons Attribution License, which permits unrestricted use, distribution, and reproduction in any medium, provided the original author and source are credited.

Funding: Supported by National Institutes of Health grant NS050524 to R. B.-G. The funders had no role in study design, data collection and analysis, decision to publish, or preparation of the manuscript.

Competing Interests: The authors have declared that no competing interests exist.

*E-mail: rbaliceg@mail.med.upenn.edu

\section{Introduction}

Congenital disorder of glycosylation, type IIc (CDG IIc), also known as leukocyte adhesion deficiency II (LAD II) or RambamHasharon syndrome (RHS), is an autosomal recessive syndrome, characterized by recurrent infections, persistent leukocytosis, severe mental retardation and slowed growth $[1,2]$. The immunodeficiency that is a hallmark of these syndromes is believed to be caused by dysregulated fucose metabolism, resulting in the absence of all fucosylated glycans on the cell surface [1,2]. The gene responsible for CDG IIc has been identified as GDP-fucose transporter (FUCT1) [3,4], which translocates GDP-fucose from the cytosol into the Golgi lumen for fucosyltransferase-catalyzed reactions during the modification of glycans.

Several animal models have been generated to study the pathogenesis of CDG IIc: $F X$ locus null mice, lacking an enzyme in the de novo GDP-fucose synthesis pathway [5], Gfr (homologous to FUCT1) null flies [6] and Fuct1 null mice [7]. Gfr null flies display Notch-like phenotypes during wing development and reduced Notch fucosylation, suggesting that Notch deficiency may be responsible for some of the developmental defects in GDG IIc patients [6]. However, despite the neurodevelopmental and cognitive dysfunction prominent in CDG IIc patients, the anatomical, cellular and molecular abnormalities within the nervous system have not been well documented, and the mechanisms underlying this and other neural phenotypes remain unexplored.
A large body of literature has demonstrated an important role for Notch-Delta signaling in neuronal and glial specification, neuronal maturation and learning and memory [8]. Specifically, in zebrafish, Notch-Delta signaling has been shown to regulate neurogenesis and gliogenesis. For instance, deficiency of Notchla as in deadly seven (des) mutants resulted in increased primary motor neuron and Mauthner neuron number [9]; deficiency of Delta A as in dla mutant caused excessive primary motor neurogenesis at the expense of secondary motor neurons, some ventral interneurons and oligodendrocytes [10,11,12]; mutation of Mind Bomb (an E3 ubiquitin ligase for Delta) as in mib resulted in a severe neurogenic phenotype together with the loss of oligodendrocytes $[12,13]$. While some studies support the involvement of Notch signaling in the balance of excitatory/ inhibitory synapses in hippocampus [14] and during synaptic plasticity [15], whether Notch-Delta signaling modulates synaptogenesis is unknown.

Here we report the genetic, cellular and molecular characterization of a zebrafish mutant slytherin $(\mathrm{sm})$. Previously, we have identified $s m$ as a synaptogenic mutant that exhibits abnormal swimming behavior, has increased primary motor neurons and aberrant neuromuscular synaptogenesis [16]. We have found that the $s m$ mutation resides in GDP-mannose 4, 6-dehydratase (GMDS), the first and rate-limiting enzyme in the fucose metabolism pathway. Because dysfunction of the same pathway is responsible for human CDG IIc, we performed cellular and molecular analyses that suggest that $s m$ has Notch-Delta dependent and independent defects, consistent with a general 
defect in protein fucosylation that affects several aspects of neural development.

\section{Materials and Methods}

\section{Zebrafish maintenance and mutants}

Zebrafish were raised and maintained under standard conditions. The sm allele was previously described [16]. The des ${ }^{b 420}$ allele was obtained from Dr. Christine Beattie, $\mathrm{Tg}$ (hsp70l:GAL4) and $\operatorname{Tg}$ (UAS:myc-notch1a-intra) [17] from Dr. Bruce Appel, and $d l a^{h i 781}$ and mib $b^{h i 904}$ alleles from Zebrafish International Resource Center, University of Oregon.

\section{Positional cloning of srn}

Genetic mapping of mutant loci was performed as described [18]. New simple sequence repeat (SSR) markers DKEY-25E12-SSR2 (forward, 5'-gcacacatgcatacgttcag-3' ${ }^{\prime}$; reverse, 5' -tcccaaagtgaaagggtgag-3') and DKEY-177P2-SSR4 (forward, 5'-cctgagggtcaggagagtaatg-3'; reverse, 5'-gaactaacactttcacaaacaccaa- $\left.3^{\prime}\right)$ were used to define the interval that contained the mutation. PCR products containing the entire ORF of gmds (accession \# NM_200489) were generated with the primers $5^{\prime}$-cggatgtgttgcatccgta-3' and 5'tcacatgaattaaacggcat- $3^{\prime}$ for both mutant and WT (WT) cDNAs, cloned into pCR 4-TOPO (Invitrogen), and sequenced for validation.

\section{RNA extraction and quantitative RT-PCR (qRT-PCR)}

RNA was extracted (20 embryos) with the RNeasy kit (Qiagen, Inc.). hes 5 was amplified with primers $5^{\prime}$-gaaagccagtggtggaaaag- $3^{\prime}$ and $5^{\prime}$-gaaagccagtggtggaaaag- $3^{\prime}$. her 4 was amplified with primers $5^{\prime}$-cctggagatgacgcttgatt- 3 ' and 5 '-cactgggcactgagacagaa- $3^{\prime}$. heyl was amplified with primers $5^{\prime}$ - gcgatacctcagctctttgg- $3^{\prime}$ and $5^{\prime}-$ ggagaggatccagctcactg- $3^{\prime}$. $\beta$-actin 1 was amplified with primers $5^{\prime}$ tgaatcccaaagccaacagagaga- $3^{\prime}$ and $5^{\prime}$-tcacgaccagctagatccagacg- $3^{\prime}$. qRT-PCR was performed with the SuperScript ${ }^{\circledR}$ III Platinum ${ }^{\circledR}$ $\mathrm{SYBR}^{\circledR}$ Green One-Step qPGR Kit w/ROX (Invitrogen) and data was analyzed with 7500 Real-Time PCR System software (Applied Biosystems) using the $2^{-\Delta \mathrm{CT}}$ method.

\section{Whole mount in situ hybridization}

gmds cDNA was cloned into pBluescript (Stratagene). The plasmid was linearized and anti-sense and sense probes were made with the Dig RNA labeling kit SP/T7 (Roche). hes5 in situ probe was generated with primers $5^{\prime}$-tggctcctgcgtatatgactgaat-3' and $5^{\prime}$ gcggetcctgcttgatgtgt- $3^{\prime}$. her4 in situ probe was generated with primers $5^{\prime}$-tctgatcctgacggagaactg- $3^{\prime}$ and $5^{\prime}$-ttcagtccatgccaatctca- $3^{\prime}$ [19]. heyl in situ probe was generated with primers $5^{\prime}$-tcaaccacagcctgtcagag- $3^{\prime}$ and $5^{\prime}$-caggggaatgctgttgaagt-3' [20]. In situ hybridization was performed as described previously [16].

\section{GDP-fucose rescue and gmds mRNA and morpholino injection}

GDP-fucose $(50 \mathrm{mM}$ in water $(\mathrm{pH}=7)$ with $0.1 \%$ phenol red as a tracer) was injected directly into $1-2$ cell stage embryos collected from crosses of $s m$ carriers. Gmds-gfp mRNAs (WT and $s m$ ) were injected into embryos from WT and $s m$ incrosses at the 1-2 cell stage at $\sim 200 \mathrm{pg}$. The morpholino antisense oligonucleotide (Gene Tools) targeting the gmds exon5-intron5 junction (CGTATGTTTGCTGACCATAAGGCGA) was injected at the $1-2$ cell stage at $\sim 4 \mathrm{ng}$.

\section{Expression of Notch1a by heat-shock induction and rescue of gmds morphant phenotypes}

To induce expression of constitutively active Notchla (Notchla intracellular domain, NICD), embryos were collected from matings of heterozygous $\mathrm{Tg}$ (hsp70l:GAL4) and $\mathrm{Tg}_{\mathrm{g}}$ (UAS:mycnotch1a-intra) adults and raised at $28.5^{\circ} \mathrm{C}$. At $11 \mathrm{hpf}$, embryos were heat-shocked at $39^{\circ} \mathrm{C}$ for 30 minutes and then returned to $28.5^{\circ} \mathrm{C}$ until the desired stage of development [21]. To determine whether NICD rescues sm phenotypes, gmds MO was injected into NICD transgenic embryos and the phenotypes were compared to NICD transgenic embryos alone, WT, sm and gmds MO embryos.

\section{DAPT treatment}

Embryos were dechorionated with forceps at $6 \mathrm{hpf}$ and placed in DAPT (N-[N-(3,5-Difluorophenacetyl-l-alanyl]-S-phenylglycine-t-butyl ester; Calbiochem) solution at $28.5^{\circ} \mathrm{C}$ until the appropriate stage, as previously described [22]. For experiments, $50 \mu \mathrm{M}$ (medium dose) and $100 \mu \mathrm{M}$ (high dose) DAPT in embryo medium containing 1\% DMSO was used. Control embryos were incubated in an equivalent concentration $(1 \%)$ of DMSO.

\section{Immunostaining, AAL staining and labeling of retinotectal projections}

Embryos were anesthetized, fixed and immunostained as described previously [16] using antibodies against SV2, Zn5, 3A10, Islet1/2, F59 (all from Developmental Studies Hybridoma Bank, Univ. of Iowa) and/or goldfish GFAP [23] (gift from Drs. S. Nona and J. Scholes, Univ. of Sussex, United Kingdom) and fluorescently conjugated secondary antibodies (Jackson Labs, Inc.). Fluorescently conjugated $\alpha$-bungarotoxin (Molecular Probes, Inc.) was used to label AChRs [16]. TUNEL staining was performed according to the manufacturer's instructions (Chemicon, Inc.). Fucosylated proteins were visualized in $48 \mathrm{hpf}$ embryos using a biotinylated fucose-specific lectin, Aleuria Aurantia lectin (AAL; $20 \mu \mathrm{g} / \mathrm{ml}$; Vector Labs; [24,25] followed by Alexa 488 conjugated strepavidin (20 $\mu \mathrm{g} / \mathrm{mL}$; Molecular Probes). The number of Zn5+ cells was counted at $20 \mu \mathrm{m}$ intervals along the rostral-caudal axis of several spinal cord hemisegments and compared statistically using Kolmogorov-Smirnov test. Retinal ganglion cell axon projections to the optic tectum were labeled as described [26].

Unless otherwise stated, each immunostaining or dye labeled figure panel is a single plane projection of a confocal z-stack of 20$1601 \mu \mathrm{m}$ thick planes (Leica TCS 4D). Presynaptic vesicles, AChR clusters and the co-localization of these two markers were measured from using interactive software (Metamorph).

\section{Results}

External phenotype, genetic cloning and mRNA rescue of slytherin

Externally, sm mutants exhibit a bent tail as early as $24 \mathrm{hpf}$, a phenotype that becomes progressively more severe (Fig. 1A), as well as a malformation of the hindbrain, which becomes apparent at $48 \mathrm{hpf}$ (Fig. 1A, brackets). The $s m$ locus was mapped between SSLP markers z49730/z14955 and z14614 on chromosome 20, with marker z10756 having no recombinants (Fig. 1B). Gmds was found to contain a $\mathrm{G}$ to $\mathrm{T}$ transversion in the nucleotide sequence that produces a nonconservative glycine $(G)$ to valine $(\mathrm{V})$ substitution of amino acid $178(\mathrm{G} 178 \mathrm{~V})$ in the short-chain dehydrogenase/reductase (SDR) domain (Fig. 1C, D, E). GMDS is highly conserved at the amino acid level; the fish and human proteins are $87 \%$ identical.

In situ hybridization showed that from 6 to $12 \mathrm{hpf}$ gmds transcripts are expressed throughout the embryo (Fig. S1A). By 24 hpf, gmds transcripts are enriched in the CNS and are also present in somites (Fig. S1B). Gmds mRNA expression is present in the CNS at 48 and $72 \mathrm{hpf}$, with transcripts more abundant in brain than spinal cord (Fig. S1C, D). Gmds mRNA is also expressed in 

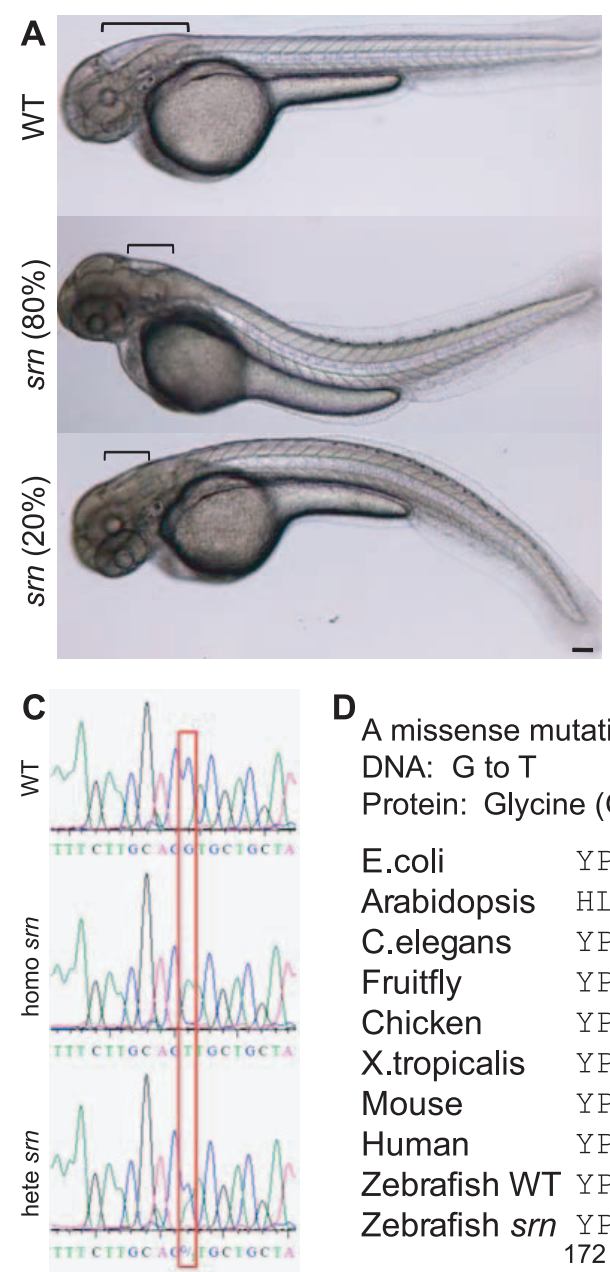

$D$

A missense mutation

DNA: $G$ to $T$

Protein: Glycine (G) to Valine (V)

E.coli YPRSPYAVAKLYAYWITVNYRE

Arabidopsis HLRSPYAASKCAAYWYTVNYRE

C.elegans YPRSPYAVAKMYGYWIVVNYRE

Fruitfly YPRSPYACAKMYGFWIVINYRE

Chicken

X.tropicalis

Mouse

Human

Zebrafish WT

Zebrafish srn

YPRSPYGAAKLYAYWIVVNFRE

YPRSPYGAAKLYAYWIVVNERE

YPRSPYGAAKLYAYWIVVNERE

YPRSPYGAAKLYAYWIVVNERE

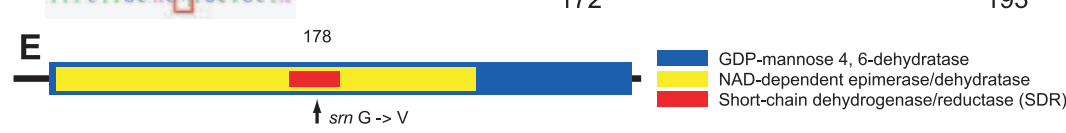

YPRSPYVAAKLYAYWIVINERE

172

Short-chain dehydrogenase/reductase (SDR)

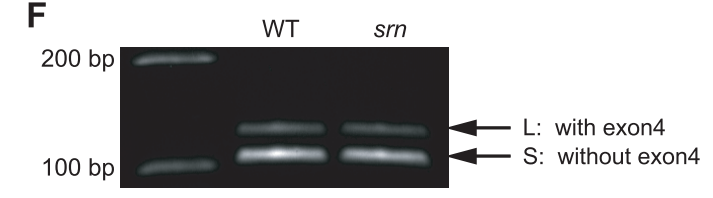

GMDS L: QTHTEGSIGATKDHMKLHYG GMDS S: QTHTEGN-.......--MKLHYG

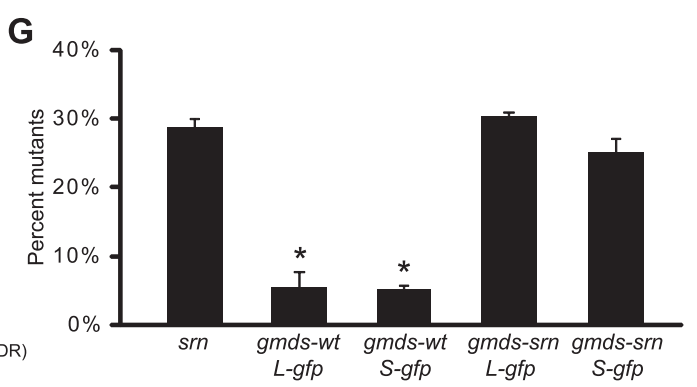

Figure 1. Slytherin external phenotype, genotype, cloning and mRNA rescue of $s r n$ mutants. A. External srn phenotypes at $48 \mathrm{hpf}$ include a bent tail ( $80 \%$ dorsal (534 embryos, 8 carrier pairs)) and aberrant hindbrain formation (brackets). Scale bar $=100 \mu \mathrm{m}$. B. Genetic and physical map of the srn locus (red arrow), including SSLP markers, number of recombinants, BAC clones and megabase positions from Ensembl Zv7. C, D. In srn, Gmds mutation is $G$ to $T$ (C, red box) resulting in a Glycine to Valine conversion (D, red box). GMDS amino acid sequence is highly conserved. E. Schematic of $s r n$ mutation in the short-chain dehydrogenase/reductase (SDR) domain of GMDS. F. Two splice variants exist in gmds mRNA, with ( $g m d s-L, 377$ aa) or without ( $g m d s-S, 370$ aa) exon 4. Gmds alternative splicing is not altered in srn mutants. G. Injection of gmds mRNA rescues srn mutants. Compared to uninjected embryos, $28.6 \pm 1.2 \%$ of embryos were mutant when scored by external phenotypes (3413 embryos, 27 carrier pairs). In embryos injected with WT gmds-gfp mRNA, the percentage of mutants scored by external phenotypes was significantly decreased, to ca. $5 \%$ (gmds-wtL-gfp 5.4 $\pm 2.5 \%, 401$ embryos, 3 carrier pairs; gmds-wtS-gfp 5.1 $\pm 0.6 \%, 587$ embryos, 4 carrier pairs; one-way ANOVA, followed by Dunn's pairwise comparison, $\mathrm{p}<0.05$ ). The percentage of embryos with mutant external phenotypes was unchanged in embryos injected with mutant $g m d s-$ gfp mRNA (gmds-srnL-gfp 30.2 $\pm 0.9 \%, 387$ embryos, 3 carrier pairs; gmds-wtS-gfp $25.1 \pm 1.9 \%, 516$ embryos, 4 carrier pairs). This mRNA rescue experiment confirms that $g m d s$ is the gene responsible for srn mutation. doi:10.1371/journal.pone.0013743.g001

the PNS at 72 hpf, including in lateral line neuromasts (data not shown; [27]).

RT-PCR analyses suggested that at least two splice variants exist in zebrafish gmds, with or without exon 4, which we name $g m d s-L$ and $g m d s-S$ respectively. Both splice variants are expressed in $s m$ mutants and WT embryos (Fig. 1F). To confirm that gmds is the gene responsible for sm phenotypes, both splice variants of the WT and mutant $g m d s \mathrm{cDNAs}$ were fused with $g f p$ and were in vitro transcribed into mRNA and were injected into 1-2 cell stage embryos collected from srn incrosses. In embryos injected with WT $g m d s-g f p$ mRNAs, $5 \%$ were mutant scored by external phenotypes compared to uninjected embryos $(29 \%)$ or embryos injected with mutant gmds-gfp mRNAs (Fig. 1G; one-way ANOVA, followed by Dunn's pairwise comparison, $\mathrm{p}<0.05)$. Moreover, when GMDS function was perturbed in WT embryos with a splice-blocking morpholino, all defects seen in sm mutants were phenocopied (see below and Fig. S3). These experiments confirm that gmds is the gene mutated in sm.

Slytherin mutants exhibit reduced protein fucosylation

GMDS is the first enzyme in the de novo fucose metabolism pathway, catalyzing the conversion of GDP-D-mannose to GDP4-keto-6-D-deoxymannose, which is further processed into GDPfucose [5] and transported into the Golgi where it is used to 
fucosylate proteins, including Selectins, Notch and many others $[5,28,29]$. Thus AAL staining for fucosylated proteins was performed in 48 hpf WT and mutant embryos (Fig. 2).

In WT embryos, AAL staining was detected in many tissues (Fig. 2A), including olfactory bulb, retina, optic tectum, hindbrain and spinal cord (Fig. 2B-E), which prompted us to examine the potential phenotypes in these structures in sm. Moreover, at neuromuscular junctions (NMJ), AAL staining co-localizes with markers for pre- and postsynaptic specializations, such as SV2 and acetylcholine receptors (AChRs) (Fig. $2 \mathrm{~K}-\mathrm{N}$ ). In contrast, AAL staining is strongly reduced in $s m$ mutants (Fig. 2F-J, O-R), consistent with analyses of cells from CDG IIc patients [3,4], and of Drosophila Gfr mutants [6]. These studies show that protein fucosylation is dramatically reduced in the CNS and other tissues in sm, consistent with a loss of function of GMDS, confirming a prediction based on the modeling of the protein crystal structure (see Fig. S2).
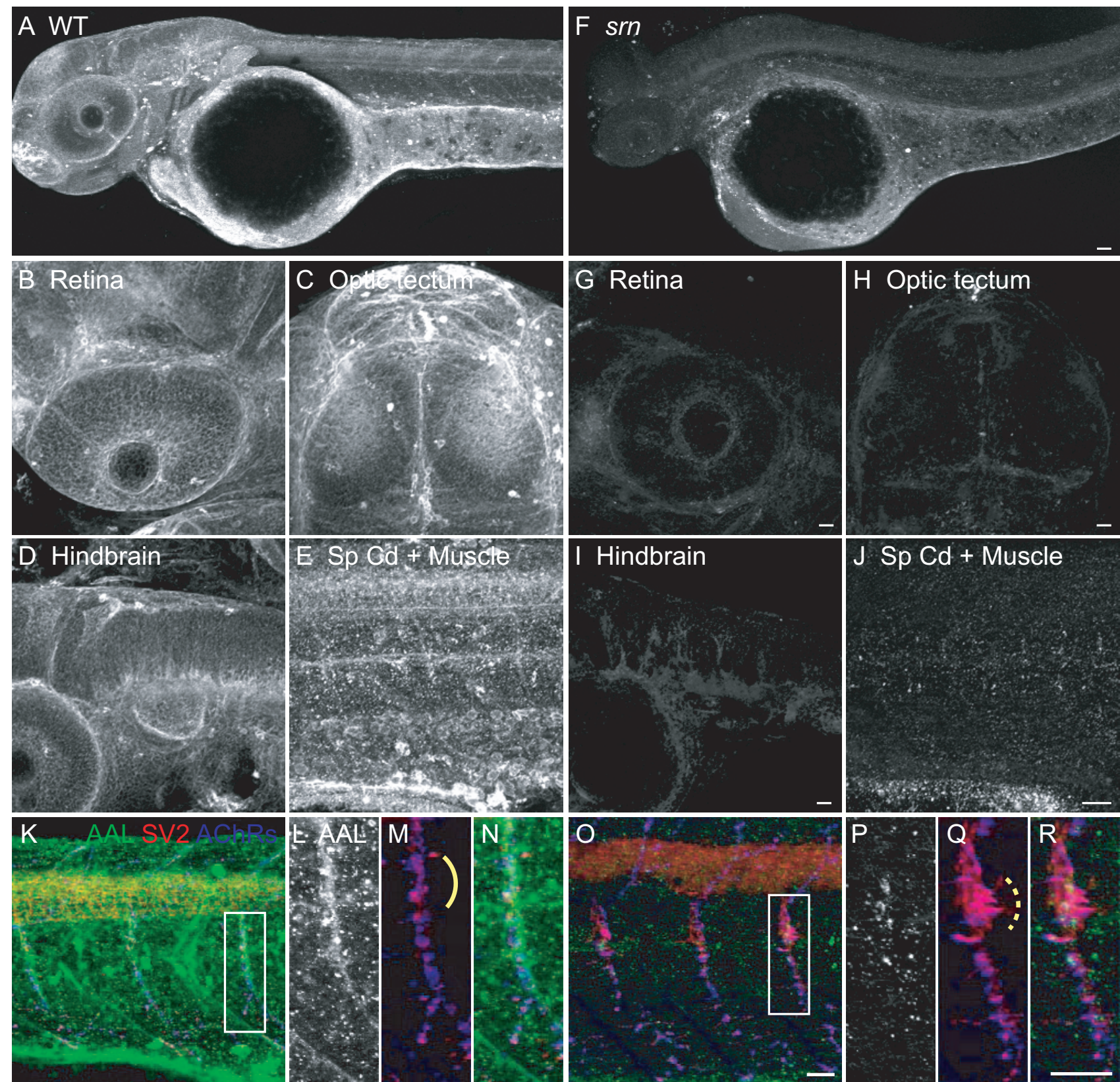

Figure 2. slytherin mutants exhibit reduced protein fucosylation as measured by AAL staining. A. AAL staining of WT embryos at $48 \mathrm{hpf}$ showed that protein fucosylation is present throughout the embryo (10-15 embryos/2-3 adult pairs for all analyses). B-E. Protein fucosylation is prominent in several neural tissues including retina (lateral view), optic tectum (dorsal view), hindbrain (lateral view), spinal cord (lateral view) and neuromuscular synapses (lateral view of axial muscle). F. Protein fucosylation is dramatically reduced in srn mutants. Scale bar $=20 \mu \mathrm{m}$. G-J. Reduced protein fucosylation in several neural tissues. Scale bar $=20 \mu \mathrm{m}$. K. Protein fucosylation at neuromuscular synapses in WT embryos at 48 hpf, as shown by the colocalization of AAL staining (green) with markers for presynaptic axons and nerve terminals (SV2, red) and postsynaptic AChR clusters ( $\alpha$-bungarotoxin, blue). L-N. Higher magnification of boxed region in K. O. Protein fucosylation is reduced at srn neuromuscular synapses. Scale bar $=20 \mu \mathrm{m}$. P-R. Higher magnification of boxed region in $\mathbf{O}$. Synapse area is significantly increased in srn mutants, e.g., at the choice point (compare dashed bracket in $\mathrm{Q}$ to solid bracket in M). Scale bar $=20 \mu \mathrm{m}$.

doi:10.1371/journal.pone.0013743.g002 


\section{Supplementation with GDP-fucose rescues slytherin phenotypes}

Since GMDS functions early in the fucose metabolism pathway, we reasoned that exogenous supply of downstream products may circumvent the genetic defect in $\mathrm{sm}$. Therefore, $50 \mathrm{mM}$ GDPfucose was injected into 1-2 cell stage embryos collected from sm incrosses. Compared to uninjected embryos, the percentage of mutant embryos, as scored by external phenotypes (Fig. 3A), was dramatically reduced in GDP-fucose injected embryos (Fig. 3B). Moreover, AAL staining was similar to that in WT embryos at 48 hpf in many if not all tissues (Fig. 3C). Detailed phenotypic analyses further showed that GDP-fucose supplementation is sufficient to rescue neural defects in sm mutants (see below and Fig. S3). These strongly suggest that the absence of GDP-fucose, as a result of GMDS dysfunction, is the cause of the sm mutant phenotypes, rather than the accumulation of the substrate, GDPmannose. Thus sm mutants display dysregulated protein fucosylation, as is seen in human CDG IIc patients, and that GDP-fucose supplementation restores fucosylation and rescues defects in sm.
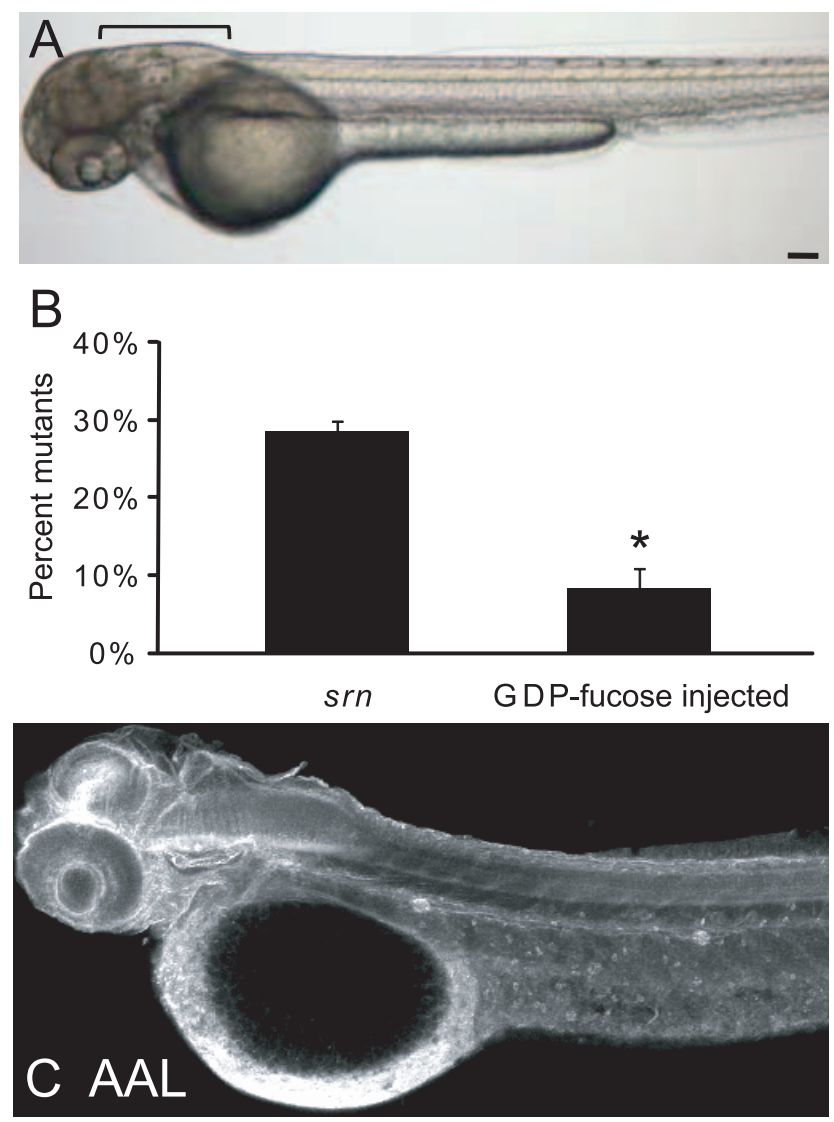

Figure 3. Supplementation with exogenous GDP-fucose rescues srn external phenotypes and restores AAL staining. A. External srn phenotypes including bent tail and aberrant hindbrain (bracket) are rescued by GDP-fucose supplementation (3 embryos). Scale bar $=100 \mu \mathrm{m}$. B. GDP-fucose injection significantly reduced the percentage of mutants from $28.6 \pm 1.2 \%$ to $8.4 \pm 2.7 \%$ (576 embryos, 4 carrier pairs; Student's test, $\mathrm{p}<0.0001)$. C. After GDP-fucose supplementation ( 2 embryos), protein fucosylation as assessed by AAL staining at $48 \mathrm{hpf}$ is rescued throughout srn embryos, to levels similar to those in WT embryos. Scale bar $=100 \mu \mathrm{m}$.

doi:10.1371/journal.pone.0013743.g003
Slytherin mutants exhibit defects in neuron and glia number, identity, patterning and axon outgrowth due to Notch-Delta signaling reduction

Our previous work suggested that sm exhibited a neurogenic phenotype, specifically an increased number of primary motor neurons [16], similar to that observed in mutants in the NotchDelta pathway. Analyses of Drosophila Gfr mutants suggested that Notch fucosylation is reduced, and that a reduction in Notch signaling might contribute to the pathogenesis in CDG IIc [6]. Therefore, we asked which if any neural defects in $s m$ were similar to those observed in mutants in the Notch-Delta pathway or in embryos treated with the $\gamma$-secretase inhibitor DAPT to reduce Notch signaling.

We compared sm phenotypes with known mutants in the NotchDelta pathway, des ${ }^{b 20}$ (deadly seven, a nonsense mutation in notch1a yielding a truncated protein; Gray et al., 2007), dla $a^{\text {hi781 }}$ (delta A, an insertion in delta $A$, predicted to result in a truncated protein [30]) and mib $^{\text {higo4 }}$ (mind bomb, an insertion in an E3 ligase that targets Delta and other proteins for ubiquitination [13], predicted to result in a truncated protein [31]). Below we describe phenotypes in each mutant in order of increasing disruption of Notch-Delta signaling.

First, we examined secondary motor neuron cell body number and patterning in the spinal cord, and axon projections in muscle using Zn5 immunostaining. In sm mutants at $48 \mathrm{hpf}$ and $72 \mathrm{hpf}$, while the number of $\mathrm{Zn} 5+$ cells is similar between sm mutant and WT embryos (Fig. S4), the patterning of these cells is aberrant. Cell bodies are clumped in sm mutants (Fig. 4A, B, second panel, dashed blue bracket), compared to evenly spaced cell bodies in WT embryos (Fig. 4A, B, top, solid blue bracket). The dorsally projecting nerve also is absent in $s m$ mutants (Fig. 4A, B, second panel, dashed pink oval), consistent with increased Zn5+ cell death [16]. des mutants do not have defects in Zn5+ cell number or patterning, but do have motor axon pathfinding errors, possibly due to aberrant formation of somite boundary (Fig. 4A; [9]). dla mutants do not have defects in Zn5+ cell number, but have similar aberrant patterning as in sm mutants, without the loss of the dorsal projecting nerve (Fig. 4A, B, dashed blue bracket and solid pink oval, respectively). mib mutants have aberrant $\mathrm{Zn} 5+$ cell number and patterning that is apparent at 48 and $72 \mathrm{hpf}$, as well as loss of the dorsal nerve.

To analyze the Zn5+ cell patterning defects quantitatively, we counted the number of Zn5+ cells at every $20 \mu \mathrm{m}$ interval along the rostral-caudal axis of several spinal cord hemisegments. This analysis showed that, while there are $3-5 \mathrm{Zn} 5+$ cells every $20 \mu \mathrm{m}$ in WT and des mutants, there are 1-9 in sm and dla, and 0-3 in mib, confirming our visual impression that patterning is aberrant (Fig. S5E-F). Moreover, while Islet $1 / 2+$ cells are dramatically increased in sm mutants at $24 \mathrm{hpf}$, consistent with increased primary motor neurons (Panzer et al., 2005), these cells are decreased at $48 \mathrm{hpf}$ and the majority of $\mathrm{Zn} 5+$ cells lack Islet $1 / 2$ expression in sm mutants (Fig. S5). As Zn5 is expressed in secondary motor neurons and is colocalized with Islet $1 / 2$ in wild type embryos, and that Islet $1 / 2$ is reduced in $\mathrm{Zn} 5+$ cells in sm, our results suggest the patterning defects in $\mathrm{Zn} 5+$ cells may be correlated with the aberrant Islet1/2 expression. There may be a defect in secondary motor neuron specification in $s m$, consistent with a role for Isletl and Islet2 in secondary motor neuron formation and axonogenesis [32].

We also found that in the spinal cord, the number of RohonBeard neurons is also significantly increased in sm mutants at 24 and $48 \mathrm{hpf}$ (Fig. S5A-B), similar to dla mutants [33], consistent with reduced Notch-Delta signaling in sm mutants. 


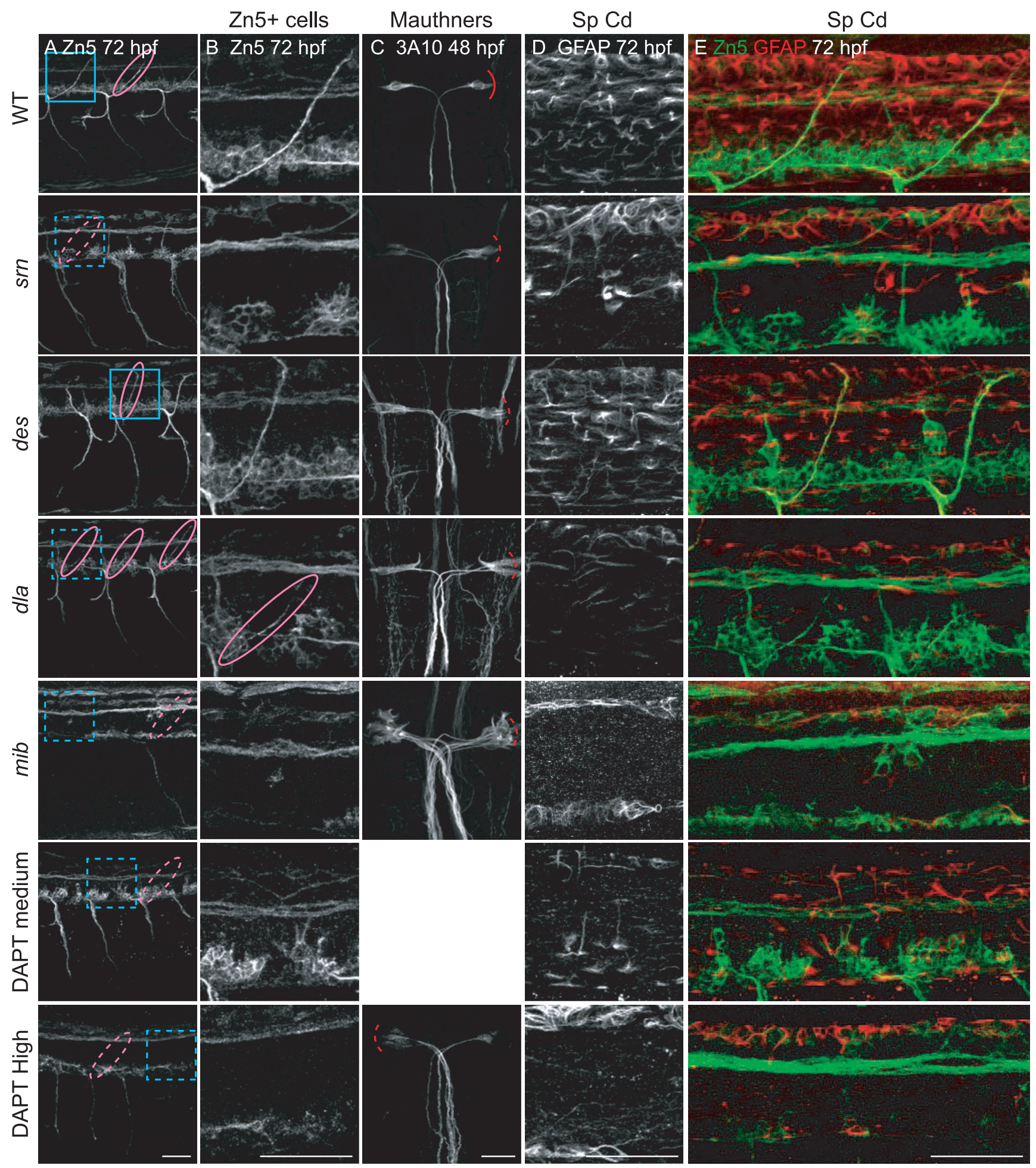

Figure 4. Reduction in Notch-Delta signaling accounts for some srn phenotypes. A, B. Secondary motor neuron cell body number and patterning assayed with Zn5 immunostaining (18 embryos/3 carrier pairs for each). B. Higher magnification of boxed region in A. At 48-72 hpf, Zn5+ cell number is similar in srn and WT (Fig. S4), but the patterning of these cells is aberrant in srn embryos. Zn5+ cells are clumped in srn mutants (dashed blue bracket) compared to WT embryos (solid blue bracket). dla mutants do not have defects in Zn5+ cell number (Fig. S4), but have aberrant Zn5+ cell patterning as in srn mutants (dashed blue bracket). mib mutants and high dose DAPT treated embryos have aberrant Zn5+ cell number (Fig. S4) and patterning (dashed blue bracket). Medium dose DAPT treated embryos show aberrant Zn5+ cell patterning defects (dashed blue bracket), without an obvious change in cell number (Fig. S4), as in srn. The dorsal projecting nerve is absent in srn mutants (dashed pink oval) compared to WT (solid pink oval), consistent with increased cell death; this nerve is present in dla and des mutants (solid pink oval); des also has other motor axon pathfinding errors. In mib mutants and high and medium dose DAPT treated embryos, the dorsal projecting nerve is absent (dashed pink oval). C. In WT embryos at $48 \mathrm{hpf}$, two Mauthner neurons are present (dorsal view of hindbrain). In srn, des, dla, mib and high dose DAPT treated embryos, Mauthner neuron number is increased (dashed red brackets), with the largest increase observed in mib (12 embryos, 3 carrier pairs for each). D. In the 
spinal cord, the number of GFAP+ glial cells is reduced in srn and dla mutants and medium dose DAPT treated embryos compared to WT and des embryos at 48-72 hpf. In mib and high dose DAPT treated embryos, a more dramatic reduction is observed. The GFAP labeling that remains in mib mutants is likely to be in Rohon-Beard neurons dorsally and secondary motor neurons ventrally and is easily separated morphologically and based on its location from glial processes, thus does not interfere with analyses of glial defects (18 embryos, 3 carrier pairs for each). E. Overlay showing both the Zn5 and GFAP staining in the spinal cord. Scale bars $=40 \mu \mathrm{m}$. doi:10.1371/journal.pone.0013743.g004

In the hindbrain and retina, similar defects in neuron number and patterning are present. In the hindbrain at $48 \mathrm{hpf}$, an increase in Mauthner neurons is observed in $s m$, des (as previously reported, Gray et al., 2001), dla and mib, with the largest increase in Mauthner neuron number observed in mib (Fig. 4C, red brackets). Moreover, neuronal patterning in the hindbrain is severely perturbed in sm and in mib (data not shown). In the retina at 72 hpf, cell number and patterning appear grossly normal in sm, des and dla, but in mib, retinal ganglion cell number is reduced (Fig. $\mathrm{S} 6 \mathrm{~A})$, probably due to increased cell death, as previously reported [34]. These data suggest that reduced Notch-Delta signaling may account for some of the CNS and PNS phenotypes observed in sm.

Because deficiencies in Notch-Delta signaling have been shown to result in reduced gliogenesis $[10,11,12,13]$, we examined glial cells in the spinal cord, hindbrain and retina with GFAP immunostaining. In the spinal cord and hindbrain, the number of GFAP+ glial cells is reduced in sm mutants compared to WT embryos at 48-72 hpf (Fig. 4D, 4E and data not shown). A similar reduction in GFAP+ glial cells is also observed in dla and mib, but not in des (Fig. 4D, 4E and data not shown). In the retina, the number of radially oriented GFAP+ Muller cells is decreased in sm and mib, but not in des or dla (Fig. S6B). These results suggest that a reduction in Notch-Delta signaling may account for the reduction in glia observed in sm mutants.

We then compared sm phenotypes with those caused by Notch signaling inhibitor DAPT, a $\gamma$-secretase inhibitor, that prevents intramembrane proteolysis of Notch and thus decreases the downstream signaling dependent on the Notch intracellular domain [22]. While high dose of DAPT treatment resulted in phenotypes resembling those seen in mib (Fig. 4 and Fig. S6), medium dose DAPT treatment closely recapitulated sm phenotypes, including the Zn5+ cell patterning defects and the reduction of GFAP+ glial cells in the spinal cord and retina (Fig. 4; Figs. S5, Fig. S6). These results substantiate the conclusion that a reduction in Notch-Delta signaling may account for the observed neural defects in sm mutants.

In order to test the synergy between sm and Notch-Delta deficiency, we initially sought to examine embryos double heterozygous for sm and mib, but these embryos did not show any obvious defects, likely because both single heterozygous embryos are haploid sufficient. We also examined embryos double homozygous for sm and mib, reasoning since Notch signaling is mostly if not completely absent in mib [13], if sm defects are also caused by Notch signaling deficiency, introducing sm into mib background would not result in addictive effects, i.e. would not be more severe then mib. Indeed, sm and mib double mutants showed reduced Zn5+ cells and GFAP+ glial cells in the spinal cord, closely resembling those seen in mib (Fig. 5). Furthermore, using the same reasoning, we tested the synergy between sm and DAPT treatment. Similarly, in DAPT high dose treated embryos, in which Notch signaling is mostly if not completely blocked, sm did not add to the defects caused by DAPT alone, i.e. DAPT treated $s m$ mutants resembled DAPT treated WT embryos showing similar reduced Zn5+ cells and GFAP+ glial cells in the spinal cord (Fig. 5). These results are consistent with the hypothesis that Notch signaling deficiency underlies the neurogenesis and gliogenesis defects in $\mathrm{sm}$.

If the observed neural defects in $\mathrm{sm}$ results from reduced Notch signaling, then overexpressing constitutively active Notch would rescue these phenotypes. We utilized transgenic lines in which a constitutively active form of Notch, Notchla intracellular domain (NICD) is overexpressed under the heat-shock promoter (Tg(hsp70l:GAL4); Tg(UAS:myc-notch1a-intra)) [17], recapitulated sm phenotypes in these embryos by morpholino knockdown of gmds transcripts, and examined whether NICD rescued the neural defects. Indeed, NICD overexpression rescued the Zn5+ cell patterning and reduced GFAP+ glial cells phenotypes in gmds morphants (Fig. 6). Moreover, NICD overexpression suppressed the increased mauthner neuron phenotype in gmds morphants (Fig. S7). These results strongly suggest that Notch signaling deficiency underlies the neurogenesis and gliogenesis defects in $\mathrm{sm}$.

To further assess whether Notch-Delta signaling is deficient in sm mutants, we examined the expression of several Notch effector genes, including hes5, her 4 and heyl as direct readout of Notch transcriptional activation, using real time quantitative RT-PCR and in situ hybridization. mib embryos display a strong reduction in Notch signaling [13] and hes5, her4 and heyl were collectively shown to be reduced in mib mutant fish and/or mice [35,36,37,38,39]. We found that, at $48 \mathrm{hpf}$, hes5, her4 and heyl expression were significantly reduced in sm mutants, similar as in mib mutants, although to a lesser extent (Fig. 7A, B). Because these data show that defects in neuron and glia number, patterning and Notch effector genes expression in srn mutants are similar to those observed in mutants in the Notch-Delta pathway, a reduction in Notch-Delta signaling caused by the lack of fucosylation accounts for these sm phenotypes.

\section{Slytherin mutants exhibit defects in neuromuscular synaptogenesis due to Notch-Delta signaling reduction}

Because sm was first identified in a screen for mutants with defects in neuromuscular synaptogenesis, we assessed the role of protein fucosylation and Notch-Delta signaling in neuromuscular synapse formation, particularly at the choice point where the first neuromuscular synapses are made [16]. Choice point neuromuscular synapse size was increased at $24 \mathrm{hpf}$ in sm, des, dla, mib and DAPT treated embryos (Fig. 8). At $48 \mathrm{hpf}$, mib and DAPT treated embryos showed no enlargement of choice point neuromuscular synapses, likely due to a reduced number of secondary motor neurons (Fig. S4). These defects are not due to defects in muscle fiber integrity or number (Fig. S8 and [16]). These results show that dysregulated protein fucosylation in $s m$ mutants resulted in an aberrant neuromuscular synaptogenesis that was phenocopied in Notch-Delta signaling deficient embryos, suggesting that NotchDelta signaling plays an important and previously unappreciated role in neuromuscular synapse formation.

\section{Slytherin mutants exhibit defects in CNS axon branching and synaptic connectivity that are independent of Notch- Delta signaling}

Phenotypic analyses showed that $s m$ has several defects that are not present in mutants in the Notch-Delta pathway des, dla or mib, or DAPT treated embryos. In the retina, while overall cellular lamination is grossly normal in srn mutants (Fig. 9E, bottom left panel), neuropil in the outer and inner plexiform layers (OPL and IPL) are dramatically altered (Fig. 9A, arrowheads and arrows). In srn mutants at 48-72 hpf, the OPL and IPL synaptic layers are disorganized, and this is not seen in des, dla or medium dose DAPT treated embryos (Fig. 9A). In mib and high dose DAPT treated 

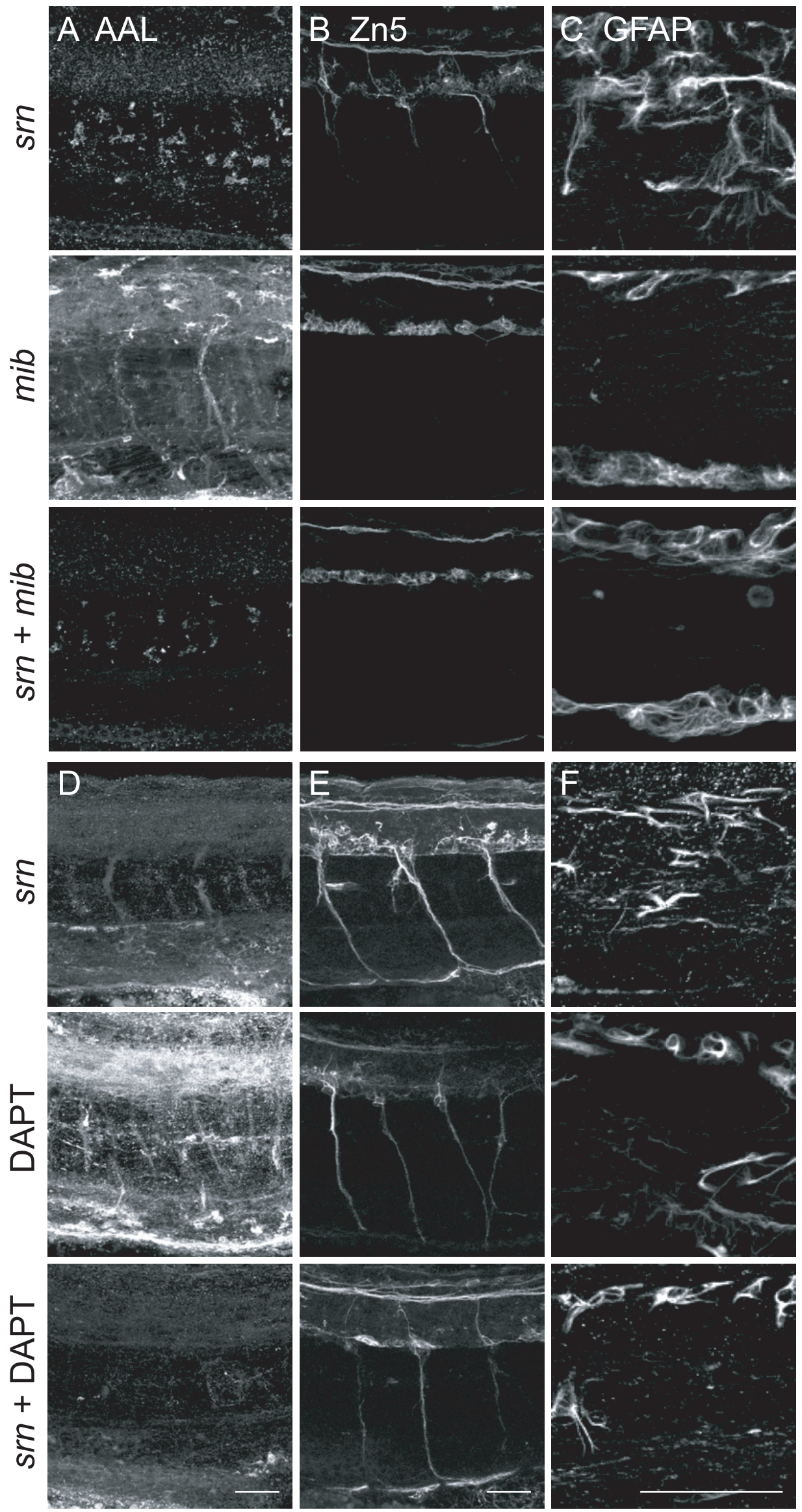

PLOS ONE I www.plosone.org 
Figure 5. mib and DAPT treatment exclude srn phenotypes. A-C. mib excludes srn phenotypes. A. AAL staining is reduced in srn and srn + mib double mutants, but not in mib. B. srn + mib double mutants showed reduction of secondary motor neurons, more severe than srn but similar to mib alone. C. $s r n+$ mib double mutants have reduced GFAP+ glia, more severe than srn, but similar to mib alone (15 embryos, 2 carrier pairs for each). Scale bar $=40 \mu \mathrm{m}$. D-F. DAPT treatment excludes srn phenotypes. D. AAL staining is reduced in srn and srn mutants treated with DAPT, but not in DAPT treated embryos. E. srn mutants treated with DAPT showed reduction of secondary motor neurons, more severe than srn but similar to DAPT treated embryos. F. srn mutants treated with DAPT showed reduction of GFAP+ glia, more severe than srn, but similar to DAPT treated WT embryos (10 embryos, 2 carrier pairs for each). Scale bar $=40 \mu \mathrm{m}$.

doi:10.1371/journal.pone.0013743.g005

embryos, retinal ganglion and other cells die, resulting in a reduction in synapses throughout the retina (Fig. 9A). Thus sm displayed unique defects in CNS synaptic connectivity that are not phenocopied by Notch signaling deficient embryos. These data are consistent with one of two possibilities. First, fucosylation of proteins other than those involved in Notch-Delta signaling may be required
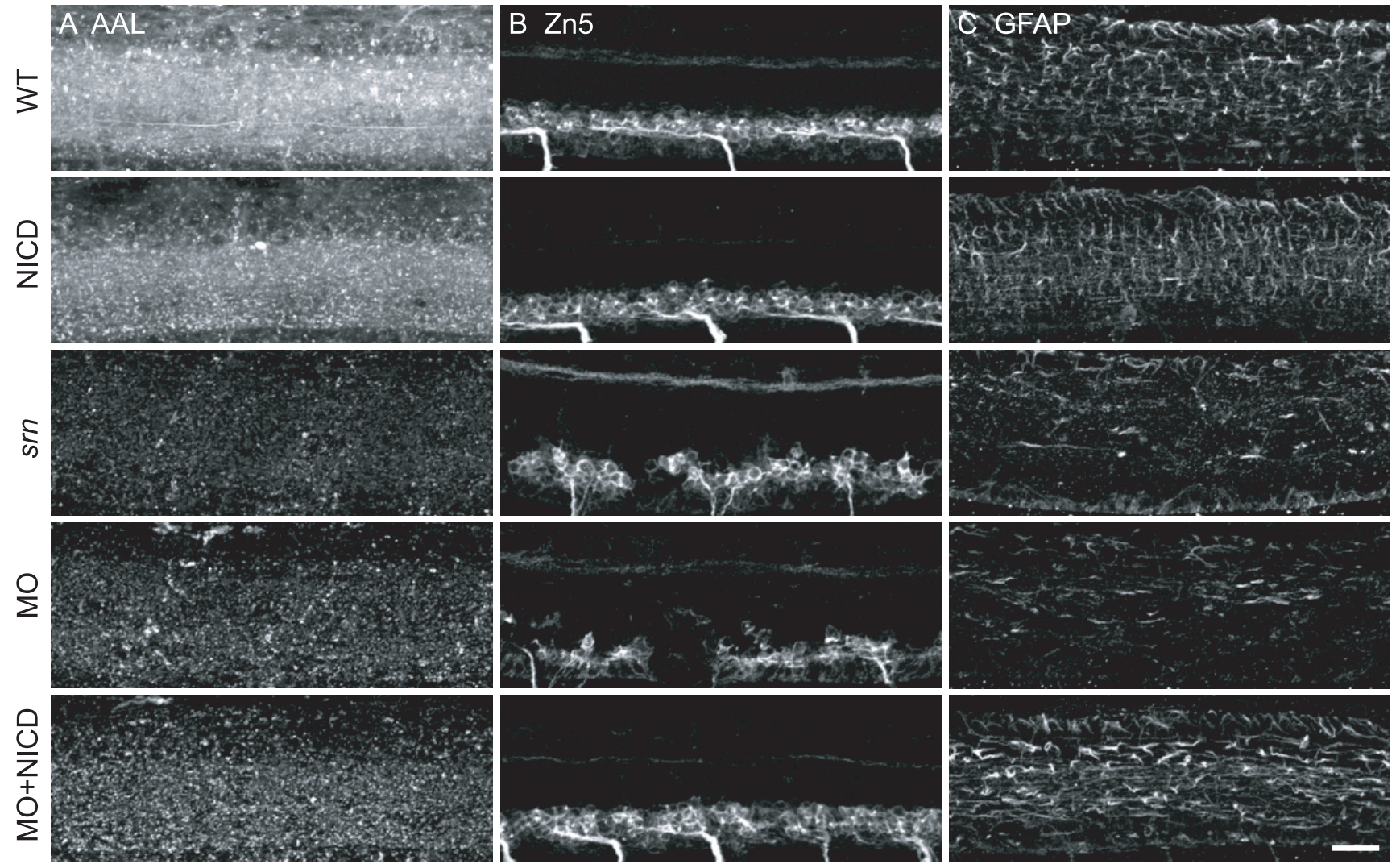

D

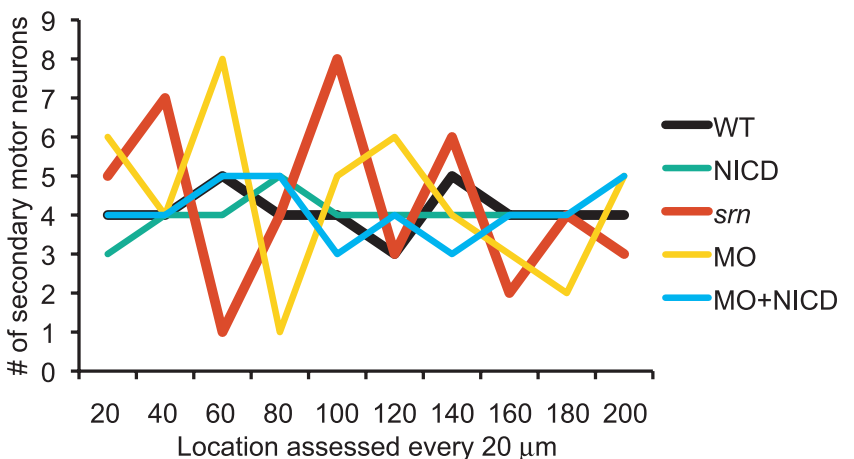

$\mathrm{E}$

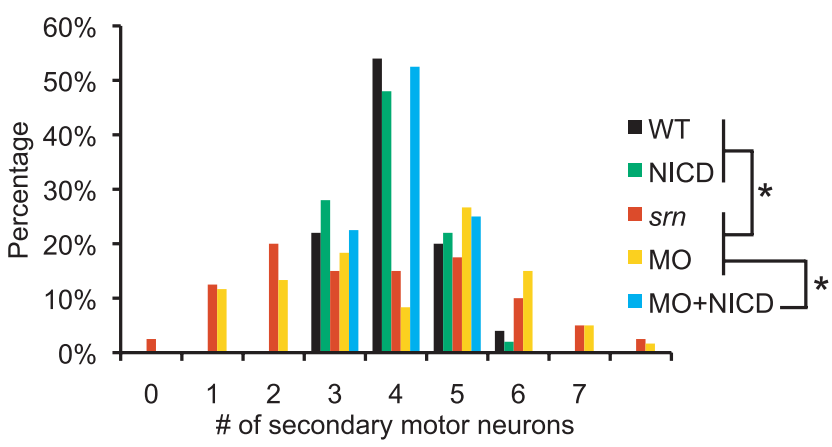

Figure 6. NICD rescues srn neuro- and gliogenesis phenotypes. A. AAL staining is reduced in srn, gmds morphants and gmds morphants overexpressing NICD, but not in WT embryos or WT embryos overexpressing NICD. B. WT and WT overexpressing NICD had normal Zn5+ cell patterning. srn and gmds MO showed Zn5+ cell patterning defects which was rescued by NICD overexpression in gmds morphants. C. WT and WT overexpressing NICD had normal GFAP+ glia cells in spinal cord. srn and gmds morphants had reduced GFAP+ glia cells, rescued by NICD overexpression in gmds morphants ( $>10$ embryos in each experiment). Scale bar $=40 \mu \mathrm{m}$. D-E. Quantification of Zn5+ cell patterning defects. There are 3-5 Zn5+ cells every $20 \mu \mathrm{m}$ in WT, WT overexpressing NICD and gmds MO overexpressing NICD; compared to 1-8 in srn and gmds MO embryos. $D$, data from a representative embryo; $E$, distribution of all embryos (4-6 embryos; Kolmogorov-Smirnov test, ${ }^{*} p<0.05$ ).

doi:10.1371/journal.pone.0013743.g006 


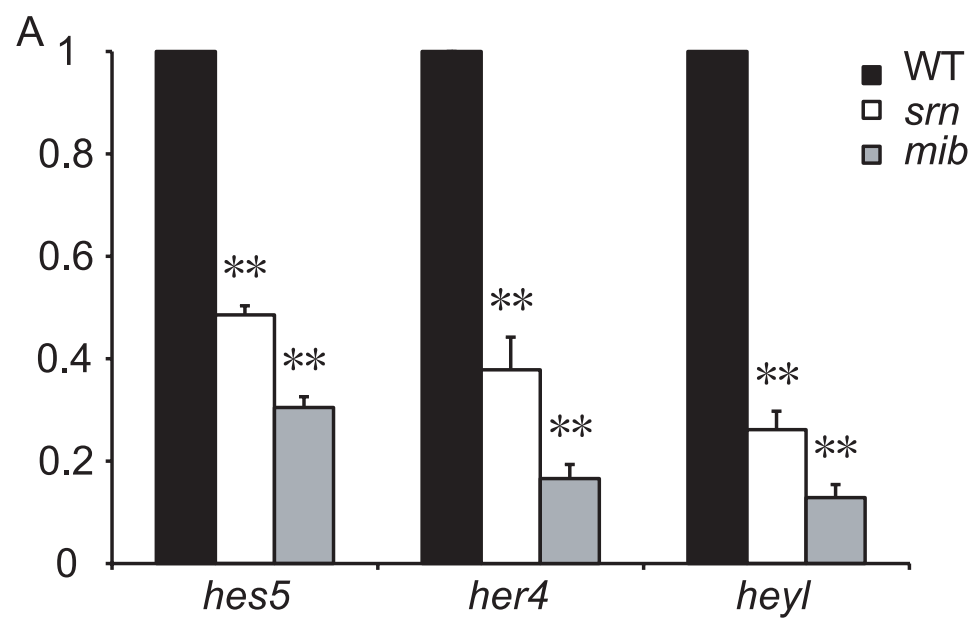

B

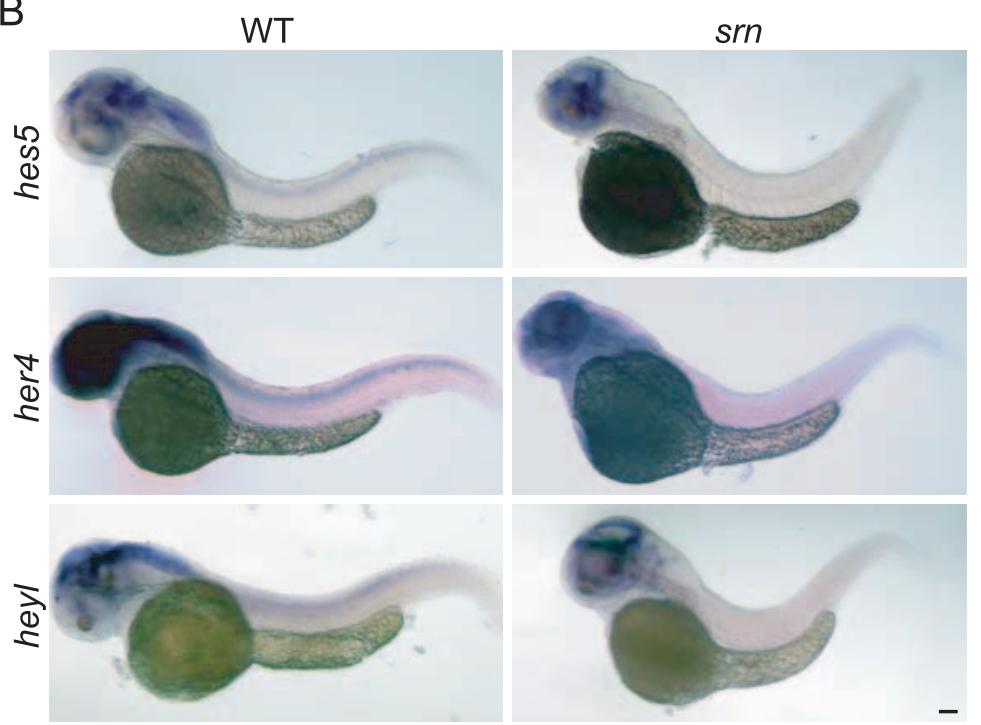

Figure 7. srn mutants showed aberrant expression of Notch responsive genes similar to mib mutants. A. qRT-PCR assessment of fold change in hes5, her4 and heyl expression in WT, srn and mib mutant embryos at 48 hpf, normalized to $\beta$-actin1. hes5, her 4 and heyl expression is dramatically reduced in $s r n$, similar to those in mib, but to a lesser extent. (3-5 experiments, 20 embryos each, one-way ANOVA, Bonferroni's Multiple Comparison Test, $\left.{ }^{* *} \mathrm{p}<0.001,{ }^{*} \mathrm{p}<0.5\right)$. B. hes5, her4 and heyl in situ hybridization at $48 \mathrm{hpf}$ confirm reduced expression in the brain and spinal cord in srn mutants compared to WT (>30 embryos for each). Scale bar $=100 \mu \mathrm{m}$. doi:10.1371/journal.pone.0013743.g007

to shape CNS synaptic connectivity. Alternatively, Notch-Delta signaling may contribute, in a specific spatio-temporal context, to these defects. Resolution of these possibilities will require identification of protein targets of srn-mediated fucosylation and exploration of their role in CNS synaptic connectivity, and/or analyses of mutants with more precise spatial and temporal disruption of Notch-Delta signaling than are currently available.

Given that AAL staining showed high levels of protein fucosylation in optic tectum (Fig. 2), we examined whether retinal ganglion cell axon outgrowth to and branching within the optic tectum was affected in $s m$ and other mutants. In sm mutants, retinal ganglion axons grow to the correct location (Fig. 9B), but their axons are aberrantly branched within the optic tectum (Fig. 9B, dashed white circle) and medial axon projections are shifted towards the midline (Fig. 9B, compare solid arrow and dashed arrow). These phenotypes are not present in des, dla or medium dose DAPT treated embryos (Fig. 9B). In mib and high dose DAPT treated embryos, the retinal ganglion cell axon projection to optic tectum is dramatically reduced due to retinal ganglion cell death (Fig. 9B). Mib and high dose DAPT treated embryos also displayed retinal ganglion axon pathfinding errors at the optic chiasm (Fig. 9B, dashed square) and decreased branching within the optic tectum (Fig. 9B, dashed white circle). Furthermore, topographic mapping analyses, in which the dorsonasal (DN) and ventrotemporal (VT) retinal ganglion cell projections were differentially labeled (Fig. 9C, D) showed that, in sm mutants, the location of the DN and VT axon projections in the optic tectum is aberrant, and that these projections overlap aberrantly dorsally and laterally (Fig. 9D). Moreover, the cellular lamination and cell viability in the optic tectum was similar between sm and WT embryos at $72 \mathrm{hpf}$ (Fig. 9E, middle left panels). These results suggest that signaling independent of the Notch-Delta pathway, but requiring protein fucosylation, modulates axon branching and synaptic patterning in the CNS.

\section{Discussion}

We report that the sm mutation causes a loss of GMDS function, leading to a severe reduction in protein fucosylation, including that of Notch among many others. Sm displays increased 

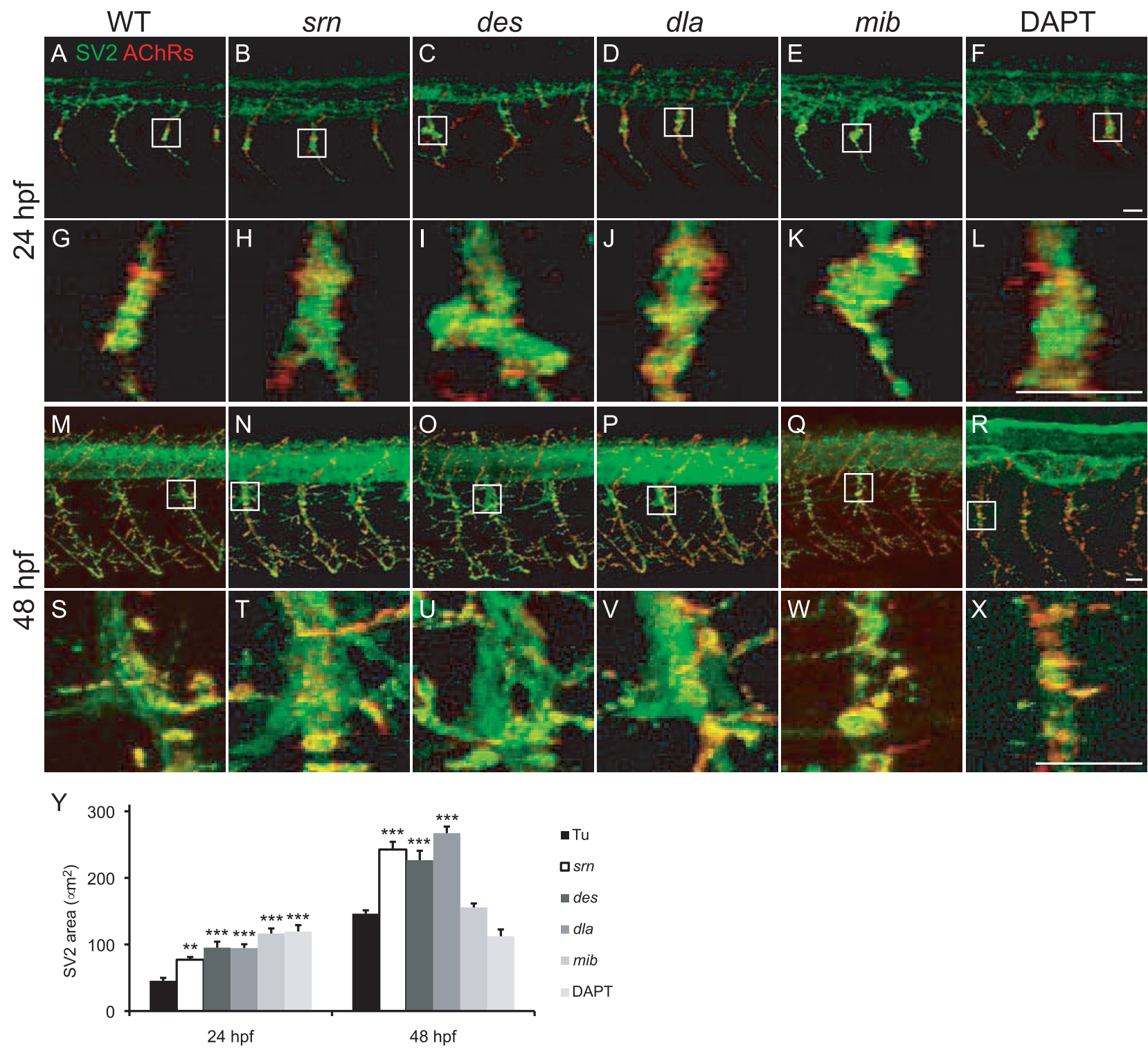

Figure 8. Slytherin mutants exhibit defects in neuromuscular synaptogenesis due in part to reduction in Notch-Delta signaling. A-X. Presynaptic terminals (green) and postsynaptic AChR clusters (red) in 24 and 48 embryos from WT $(A, G, M, S)$, srn $(B, H, N, T)$, des $(C, I, O, U)$, dla $(D, J$, $\mathrm{P}, \mathrm{V})$, mib $(\mathrm{E}, \mathrm{K}, \mathrm{Q}, \mathrm{W})$ and DAPT treated embryos $(\mathrm{F}, \mathrm{L}, \mathrm{R}, \mathrm{X})$. Boxed regions are shown at higher magnification at $24(\mathrm{G}-\mathrm{L})$ and $48 \mathrm{hpf}(\mathrm{S}-\mathrm{X} ; 3$ hemisegments in each of 20 embryos, 3 carrier pairs for each). Scale bar $=20 \mu \mathrm{m}$. Y. Presynaptic terminal, axon and synapse area at the choice point was significantly increased in all mutants, except in mib and DAPT treated embryos at $48 \mathrm{hpf}$, compared to WT (one-way ANOVA, Bonferroni's Multiple Comparison Test, ${ }^{* *} \mathrm{p}<0.01,{ }^{* * *} \mathrm{p}<0.001$ ).

doi:10.1371/journal.pone.0013743.g008

neurogenesis, decreased gliogenesis, increased neuronal cell death, abnormal neuronal patterning, abnormal axon arborization, and abnormal neuromuscular and CNS synaptic connectivity, indicating that protein fucosylation plays an important role in several aspects of neural development.

Notch-Delta signaling reduction underlies some but not all srn neural phenotypes

Our results suggest that both Notch-dependent and -independent mechanisms contribute to the neural phenotypes observed in sm. Sm mutants showed reduced Notch transcriptional activity, as assayed by hes5, her 4 and heyl expression, increased primary motor neuron, Rohon-Beard neuron and Mauthner neuron number, decreased gliogenesis and abnormal neural patterning. These defects are phenocopied by mutants in the Notch-Delta pathway and in embryos with reduced Notch signaling. That mib and Notch signaling inhibition by DAPT occlude $s m$ defects, and that NICD overexpression rescues these $s i n$ phenotypes, strongly suggest that the dysregulated fucosylation of proteins in the Notch-Delta pathway accounts for these prominent neural defects in sm mutants. While the lack of anti-zebrafish Notch antibodies prevented direct analysis of Notch fucosylation, Notch is known to be fucosylated, and other proteins in the Notch-Delta pathway, including Delta, Serrate and Jagged, contain consensus sequence(s) for O-linked fucose modification $[40,41,42]$. Notch is also Nfucosylated, in which fucose is added to N-linked glycan side chains $[6,43,44]$. Notch $\mathrm{O}$ - and $\mathrm{N}$-fucosylation has been shown to be reduced in the Drosophila Gfr null [6]. It thus seems highly 

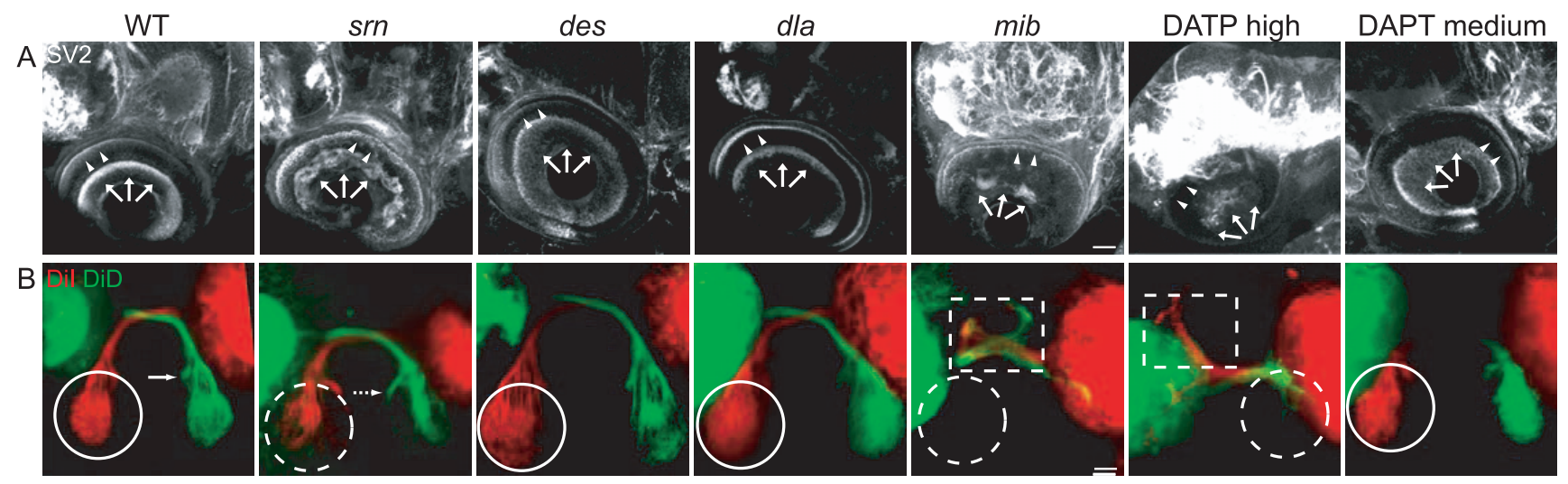

C
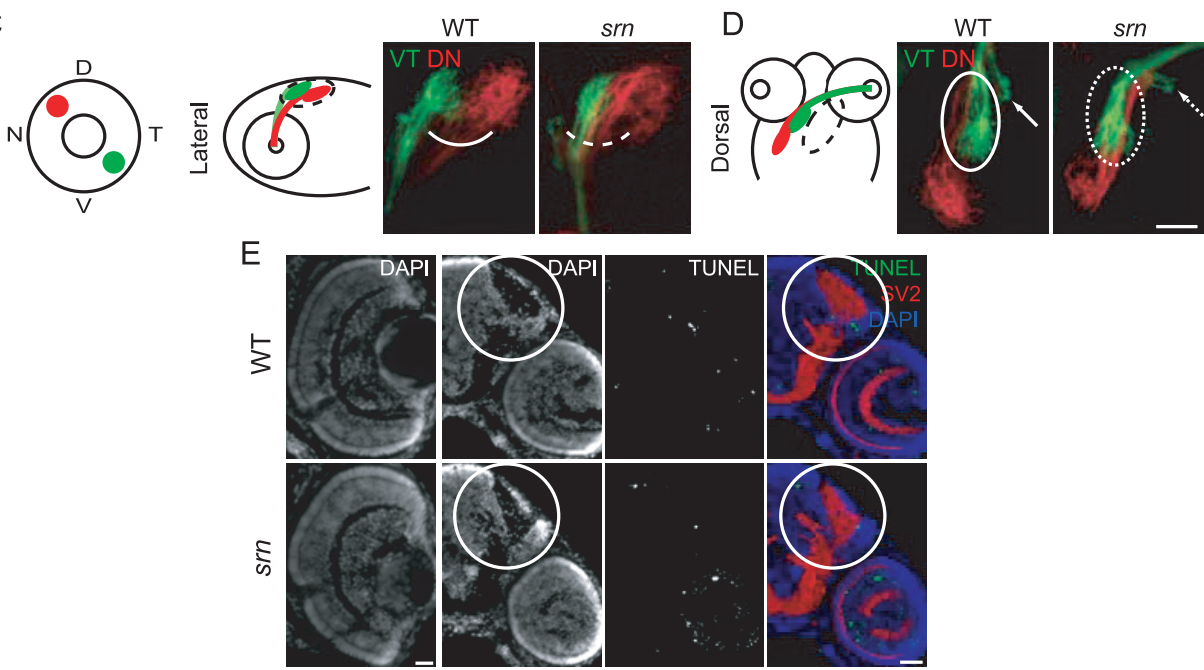

Figure 9. Slytherin mutants exhibit defects in axon branching and CNS synaptic connectivity that are independent of Notch-Delta signaling. A. In srn mutants at $72 \mathrm{hpf}$, the OPL (arrowheads) and IPL (arrows) are disorganized; this is not seen in des or dla mutants. In mib mutants, retinal ganglion and other cells die, resulting in decreased retina neuropil (rightmost panel; 8 embryos, 2 carrier pairs for each). Scale bar $=20 \mu \mathrm{m}$. B. In srn mutants, retinal ganglion cell axons grow out to the optic chiasm and to optic tectum, but axon branches are aberrantly distributed within tectum (dashed white circle) and medial axon projections are shifted towards the midline (compare solid arrow and dashed arrow). Virtually all retina was dye labeled, and the labeling pattern was consistent across experiments, thus these defecs aren't due to incomplete dye uptake or labeling in srn mutants. These phenotypes are not present in des or dla mutants, and are also different from mib mutants, in which retinal ganglion cell axonal projections to optic tectum are dramatically reduced, as a consequence of retinal ganglion cell death. Mib mutants also displayed axon pathfinding errors at the optic chiasm; axons branched anterior to the optic chiasm (dashed square), while branching within tectum was dramatically reduced (dashed white circle; 15 embryos, 3 carrier pairs for each). Scale bar $=20 \mu \mathrm{m}$. C, D. Topographic mapping of axon projections to optic tectum; dorsonasal (DN) and ventrotemporal (VT) axons were with Dil or DiD. DN and VT axon projections within tectum are aberrant in srn mutants, as is overlap dorsally (C) and laterally (D; 8 embryos, 2 carrier pairs for each). Scale bar $=20 \mu \mathrm{m}$. E. In retina and optic tectum (white circle), the overall cellular lamination pattern as assessed by DAPI staining is grossly normal in srn mutants (compare bottom left panels, WT and srn). TUNEL staining showed that increased cell death was observed in the retina of srn mutants compared to WT embryos at 72 hpf; no difference in cell death in the optic tectum was observed in srn mutants compared to WT embryos at $72 \mathrm{hpf}$ (color overlay, right most panels; 2-3 embryos, 1 carrier pair). Scale bar $=20 \mu \mathrm{m}$.

doi:10.1371/journal.pone.0013743.g009

likely that the fucosylation of proteins in the Notch-Delta pathway is aberrant in sm mutants and that this accounts for some, but not all, sm neural phenotypes.

Interestingly, there is a hierarchy in the spectrum of phenotypes among srn and mutant in the Notch-Delta pathway. Phenotypes in des, except for the axon pathfinding errors, are weaker than those in dla, and both of these are weaker than srn. This is consistent with the hypothesis that many Notch-Delta factors, including Notch, Delta, Serrate and Jagged, require proper protein fucosylation and compromised fucosylation of these proteins may account for the wider spectrum of defects seen in srn. Mib mutants also displayed a wide range of defects, not seen in the other three mutants, both due to the fact that mib regulates a large spectrum of Notch signaling, as it interacts with various Notch ligands and is broadly required for Notch signaling in many tissues, and also it interacts with a number of proteins besides Delta and may serve as an integrator of multiple neuronal developmental pathways [45].

Moreover, our observation that sm and mutants in the NotchDelta pathway have increased neuromuscular synapses supports a previously underappreciated role for Notch-Delta signaling during synaptogenesis. Because primary motor neuron number is increased in $s m$, it is difficult to separate direct effects of NotchDelta signaling on presynaptic differentiation from indirect effects on neurogenesis. The total number of motor neurons innervating trunk muscles actually decreases due to secondary motor neuron cell death [16], while the increase in neuromuscular synapse number and size persists. This strongly suggests that Notch-Delta 
signaling plays a role in synaptogenesis, independent of its role in neurogenesis.

Recent work has shown that reduced protein fucosylation, as a result of gmds mutation in twohead (twd) mutants, results in defects in the migration of vagus motor neuron progenitors [46]. However, they argued that Notch signaling is unaltered, based on several lines of evidence. First, they concluded from semi-quantitative RTPCR analyses, that expression of her4, a downstream effector in the Notch pathway, was unchanged, but their data suggests that her4 expression may indeed be decreased. On the other hand, we show using quantitative RT-PCR in our Fig. 7 that her 4 is reduced in sm mutants. Second, Ohata et al. analyzed motor neuron number and patterning by in situ for islet 1 and islet 2 and concluded that motor neuron number and patterning are unaltered in twd. On the other hand, we show that the number of neurons assayed by islet 1 and islet2 in situ [16] and by islet $1 / 2$ immunostaining at $24 \mathrm{hpf}$ (Fig. S5) is increased in sm mutants. Thus, detailed analyses of neural and glial phenotypes and analyses of additional Notch target genes in twd mutants may help resolve this apparent discrepancy.

Previous work suggested that Fringe, a glycosyltransferase that glycosylates specific sites on the Notch extracellular domain during its intracellular processing, modulates Notch activity $[29,47,48,49]$. In sm, both O- and $\mathrm{N}$-fucosylation are compromised due to reduced production of fucose moieties. Fringe acts one step downstream of O-fucosylation, adding N-glycans onto fucosylated sites. We speculate that Fringe loss of function may result in similar, but milder, deficits than in sm mutants. Indeed, recent work suggested that lunatic fringe $(L f n g)$, a known modifier of Notch, promotes the lateral inhibition of neurogenesis, that $L f n g$ loss of function by morpholino knockdown leads to increased expression of proneural genes and increased neurogenesis, and that transgenic overexpression of $L f n g$ decreases neurogenesis [50]. These observations are consistent with our results, and further support our conclusion that dysregulated glycosylation of Notch and its ligands results in Notch signaling deficiency and leads to increased neurogenesis.

While deficiencies in Notch-Delta signaling underlie some sm phenotypes, other sm phenotypes are likely to be independent of this pathway. Srn mutants exhibit prominent defects in retinotectal connectivity that are quite different from those observed in mutants in the Notch-Delta pathway such as des and dla in which no defects in retinotectal axon branching are observed, and from the dramatic reduction in retinal ganglion cell number and axon pathfinding observed in mib. We present several lines of evidence that support the conclusion that some, but clearly not all, of the mechanisms underlying the neural phenotypes in sm are Notchdependent. Future work will focus on identifying the fucosylated proteins that mediate the neural deficits that are independent of Notch-Delta signaling.

It seems likely that the regulation of Notch signaling by fucosylation is context dependent, i.e. different aspects of neural development require specific types and extent of fucosylation and other modifications of Notch receptors and/or ligands, in a particular spatiotemporal fashion. Our results do not completely rule out the possibility that Notch signaling may contribute, in a specific spatiotemporal context, to the synaptic defects and retinal ganglion cell arborization defects in sm, and this will only be resolved once the relevant fucosylation targets are identified.

\section{Srn as a zebrafish model for congenital disorders of glycosylation}

Over the last decade, a large number of human genetic diseases with aberrant glycoprotein synthesis have been identified and grouped as congenital disorders of glycosylation (CDG). Since glycosylation is essential for the function of many proteins, it is not surprising that disruption of glycosylation can lead to severe, multisystemic phenotypes, including neurodevelopmental and cognitive disorders. In sm mutants, the gmds mutation largely abolishes the synthesis of GDP-fucose, resulting in reduction or elimination of both O-linked and N-linked fucosylation of Notch and many other proteins. Thus it is possible that disruption of $\mathrm{O}-$ as well as $\mathrm{N}$ linked glycosylation of Notch and other proteins contributes to CDG IIc pathogenesis, although this has not been examined extensively in humans.

There are several reports of neural deficits in CDGIIc patients, including severe mental retardation, microcephaly, cortical atrophy, seizures, psychomotor retardation and hypotonia $[2,4,51]$. These clinical observations are consistent with the GNS and PNS cellular phenotypes observed in sm. Giving the advantage of performing imaging, genetic and pharmacological manipulations in zebrafish, sm will be a useful tool to guide future analyses in human CDG IIc patients and contribute to a better understanding of the mechanisms responsible for this devastating disorder that affects nervous system and other organ development.

\section{Supporting Information}

Figure S1 Gmds mRNA localization by in situ hybridization in wild type zebrafish embryos from 12 to $72 \mathrm{hpf}$. In situ hybridization was performed as described previously (Panzer et al., 2005), with anti-sense (A-E) gmds probe; sense probe was used as a control (F). Several hundred embryos from several carrier pairs were used from 6 to $72 \mathrm{hpf}$. A. From 6 to $12 \mathrm{hpf}$, gmds transcripts are expressed throughout the embryo. B. By 24 hpf, gmds transcripts are highly expressed in the CNS and are also expressed in somites at lower levels. C, D. Gmds mRNA expression is present in the CNS at $48(\mathrm{C})$ and $72(\mathrm{D}) \mathrm{hpf}$, with transcripts more abundant in brain than spinal cord.

Found at: doi:10.1371/journal.pone.0013743.s001 (2.49 MB EPS)

Figure S2 Modeling of zebrafish GMDS protein structure. Because of the high degree of amino acid sequence conservation between zebrafish and human GMDS, we reasoned that it would be informative to superimpose the zebrafish GMDS sequence onto the human GMDS crystal structure; this was done using a search of the using a search of the Protein Data Bank database (www.pdb. org) and MODELLER and PYMOL software. The wild type (brown rods) and srn (blue rods) primary amino acid sequence was modeled onto the human GMDS protein crystal structure. A: As in the srn mutation, Valine was substituted for Glycine at residue 178 and an energy minimization calculation was performed. When the srn mutation is present, the Valine deforms a nearby Glutamate residue, Glu155. This change is predicted to push away the substrate GDP-manose, resulting in loss of function. B: To understand how the movement of Glu155 could affect surrounding amino acids, the wild type structure (brown sticks) was examined in more detail. Three ordered $\mathrm{H} 2 \mathrm{O}$ molecules exist between the negatively charged group on Glu155 and the negatively charged phosphate group on GDP. The bond lengths between water oxygens and phosphate or carboxylic acid oxygens are appropriate to form hydrogen bonds to coordinate GDP to Glu155.

Found at: doi:10.1371/journal.pone.0013743.s002 (0.83 MB EPS)

Figure S3 GDP-fucose rescue of srn and morpholino knockdown of gmds. A. RT-PCR showed $>80 \%$ of gmds transcript was mis-spliced after gmds morpholino $(4 \mathrm{ng})$ injection. B-E. External phenotypes in srn and gmds morphants (E) include tail bend (compare B, wild type with $\mathrm{C}$, srn) which is rescued after GDP- 
fucose supplementation (D). F-I. srn (G) and gmds morphants (I) showed reduced AAL staining compared to wild type (F) which is rescued after GDP-fucose supplementation (H). J-M. srn $(\mathbf{K})$ and gmds morphants $(\mathbf{M})$ showed increased Mauthner neuron number compared to wild type $(\mathrm{J})$ a phenotype that is rescued after GDPfucose supplementation (L). N-Q. srn $(\mathrm{O})$ and gmds morphants (Q) showed reduced GFAP+ glia in the spinal cord compared to wild type $(\mathrm{N})$, a phenotype that is rescued after GDP-fucose supplementation $(\mathrm{P})$. R-U. srn $(\mathrm{S})$ and gmds morphants $(\mathrm{U})$ showed increased neuromuscular synapses compared to wild type (R), a phenotype that is rescued after GDP-fucose supplementation (T). Scale bar $=40 \mu \mathrm{m}$. In each experiment, at least $10 \mathrm{srn}$, normal siblings, gmds morphants or GDP-fucose rescued srn mutant embryos were assessed at $48 \mathrm{hpf}$. These results show that GDP-fucose rescues external and neural defects in srn mutants and that gmds knockdown by morpholino phenocopies srn phenotypes. Together, these further support the conclusions that gmds is the gene mutated in srn, that the fucose metabolism pathway is deficient in srn mutants, and that the resulting lack GDP-fucose is the cause of the srn mutant phenotypes, rather than the accumulation of the substrate, GDP-mannose.

Found at: doi:10.1371/journal.pone.0013743.s003 (6.55 MB EPS)

Figure S4 Zn5+ cell number is reduced in mib but not srn, des or dla compared to wild type embryos. The number of $\mathrm{Zn} 5+$ cells was counted from embryos at 48 and 72 hpf after immunostaining with Zn5 antibody and confocal reconstruction of the motor neuron pool. At $48 \mathrm{hpf}, \mathrm{Zn} 5+$ cell number per hemisegment was

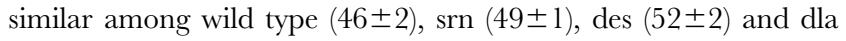
$(46 \pm 2)$ embryos, and is significantly reduced in mib mutant embryos $(27 \pm 2$ ) (1 hemisegment in each of 6-9 $48 \mathrm{hpf}$ embryos counted of each genotype; one-way ANOVA, Bonferroni's Multiple Comparison Test, only mib is significantly different compared to other mutants and wild type, $\mathrm{p}<0.001)$. At $72 \mathrm{hpf}$, Zn5+ cell number per hemisegment was similar among wild type

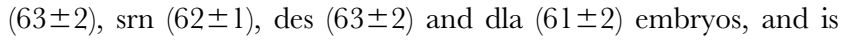
significantly reduced in mib mutant embryos (36 \pm 2$)$ (1 hemisegment in each of 6-10 $72 \mathrm{hpf}$ embryos counted of each genotype; one-way ANOVA, Bonferroni's Multiple Comparison Test; only mib is significantly different compared to other mutants and wild type, $* \mathrm{p}<0.001)$.

Found at: doi:10.1371/journal.pone.0013743.s004 (0.37 MB EPS)

Figure S5 Immunostaining of $\mathrm{Zn} 5$, Islet1/2 and GFAP, and quantification of Zn5+ cell patterning defects. A. At $24 \mathrm{hpf}$, Isletl/ 2 staining is increased in srn mutants compared to WT embryos, in primary motor neurons and Rohon-Beard neurons, identified based on their morphology and location in the spinal cord. Dashed lines indicate segment boundaries. B. At $48 \mathrm{hpf}$, in WT embryos, $\mathrm{Zn} 5+$ cells are also Islet $1 / 2+$. In srn mutants, Islet $1 / 2$ expression is reduced and majority of Zn5+ cells are not Islet1/2+. C. Zn5 and GFAP immunostaining in WT and srn mutants at $48 \mathrm{hpf}$, showing the spatial relationship between these markers. D. GFAP staining in HuC:GFP embryos at $48 \mathrm{hpf}$, showing the spatial relationship between neuron cell bodies and GFAP+ processes in the spinal cord. Scale bar $=40 \mu \mathrm{m}$. E-H. Quantification of Zn5+ cell patterning defects. There are $3-5 \mathrm{Zn} 5+$ cell at every $20 \mu \mathrm{m}$ interval in WT and des mutants, 1-9 in srn, dla mutants and medium dose DAPT treated embryos, and $0-3$ in mib and high dose DAPT treated embryos, consistent with clumping and gaps in the spinal cord. Data from a representative embryos is shown in $\mathrm{E}$ and $\mathrm{G}$. The distribution of all embryos is shown in $\mathrm{F}$ and $\mathrm{H}$ (4-9 embryos for each; Kolmogorov-Smirnov test, $\mathrm{p}<0.05$ ).
Found at: doi:10.1371/journal.pone.0013743.s005 (5.80 MB EPS)

Figure S6 Reduction in Notch-Delta signaling accounts for some srn phenotypes in the retina. A. Retina patterning was examined with immunostaining using antibody Zn5 at $72 \mathrm{hpf}$. Retina cell patterning appears grossly normal in srn, des, dla and medium dose DAPT treated embryos, but in mib and high does DAPT treated embryos retinal ganglion cell number is reduced, probably due to increased cell death, as previously reported (Bernardos et al., 2005) (8 embryos, 2 carrier pairs were examined). Scale bar $=40 \mu \mathrm{m}$. B. Glial cells in the retina were examined after immunostaining with anti-GFAP antibody. In the retina, the number of radially oriented GFAP+ Muller cells is decreased in srn and mib and medium dose DAPT treated embryos, but not in des or dla (8 embryos, 2 carrier pairs were examined). Scale bar $=40 \mu \mathrm{m}$. These results suggest that a reduction in Notch-Delta signaling may account for the glial defects observed in srn mutants.

Found at: doi:10.1371/journal.pone.0013743.s006 (2.16 MB EPS)

Figure S7 NICD overexpression suppresses the increased Mauthner neuron phenotype in gmds morphants. A. AAL staining is reduced in gmds morphants overexpressing NICD, but not in WT embryos or WT embryos overexpressing NICD. B. WT embryos have a pair of Mauthner neurons. WT overexpressing NICD showed dramatic hindbrain patterning defects, resulting in an almost complete loss of Mauthner neurons. gmds morphants overexpressing NICD showed similar reduction of Mauthner neurons. This result suggests NICD overexpression suppresses the increase of Mauthner neurons observed in gmds morphants and thus supports our conclusion that reduction of Notch-Delta signaling in srn mutants is responsible for the neurogenesis defects (3-4 embryos assessed for each manipulation). Scale bar $=20 \mu \mathrm{m}$. Found at: doi:10.1371/journal.pone.0013743.s007 (2.95 MB EPS)

Figure S8 Muscle patterning is grossly normal in srn mutants. Slow muscle fibers were examined with F59 antibody and glia cells in the spinal cord were examined with GFAP antibody. While there is an obvious reduction of GFAP+ glia cells in the spinal cord in srn mutants, the patterning of slow muscle fibers is similar in srn and wild type embryos at $48 \mathrm{hpf}$. Previous work showed that fast muscle fiber number and patterning are unaltered in srn compared to wild type embryos at 48 hpf (Panzer et al., 2005; 3 embryos, 1 carrier pair were examined). Scale bar $=200 \mu \mathrm{m}$. Found at: doi:10.1371/journal.pone.0013743.s008 (1.86 MB EPS)

\section{Acknowledgments}

We thank Dr. Christine Beattie for providing the deadly seven mutant line, Dr. Bruce Appel for providing the $T g(h s p 70 l: G A L 4)$ and $\operatorname{Tg}\left(U A S: m y c^{-}\right.$ notch1a-intra) transgenic lines, Dr. Chi-Bin Chien for help with analyses of retinal ganglion cell axon targeting and branching, Drs. Sam Nona and John Scholes for providing the goldfish GFAP antibody, Ethan Hughes for help with quantification of neuromuscular synapses, Mrs. Marion Scott for technical assistance, and members of the Balice-Gordon lab for helpful discussions.

\section{Author Contributions}

Conceived and designed the experiments: YS RBG. Performed the experiments: YS JRW PCS JAP AK RGG GBW. Analyzed the data: YS ES RBG. Wrote the paper: YS RBG. 


\section{References}

1. Becker DJ, Lowe JB (1999) Leukocyte adhesion deficiency type II. Biochim Biophys Acta 1455: 193-204.

2. Etzioni A, Sturla L, Antonellis A, Green ED, Gershoni-Baruch R, et al. (2002) Leukocyte adhesion deficiency (LAD) type II/carbohydrate deficient glycoprotein (CDG) IIc founder effect and genotype/phenotype correlation. Am J Med Genet 110: 131-135.

3. Luhn K, Wild MK, Eckhardt M, Gerardy-Schahn R, Vestweber D (2001) The gene defective in leukocyte adhesion deficiency II encodes a putative GDPfucose transporter. Nat Genet 28: 69-72.

4. Lubke T, Marquardt T, Etzioni A, Hartmann E, von Figura K, et al. (2001) Complementation cloning identifies CDG-IIc, a new type of congenital disorders of glycosylation, as a GDP-fucose transporter deficiency. Nat Genet 28: 73-76.

5. Smith PL, Myers JT, Rogers CE, Zhou L, Petryniak B, et al. (2002) Conditional control of selectin ligand expression and global fucosylation events in mice with a targeted mutation at the FX locus. J Cell Biol 158: 801-815.

6. Ishikawa HO, Higashi S, Ayukawa T, Sasamura T, Kitagawa M, et al. (2005) Notch deficiency implicated in the pathogenesis of congenital disorder of glycosylation IIc. Proc Natl Acad Sci U S A 102: 18532-18537.

7. Hellbusch CG, Sperandio M, Frommhold D, Yakubenia S, Wild MK, et al. (2007) Golgi GDP-fucose transporter-deficient mice mimic congenital disorder of glycosylation IIc/leukocyte adhesion deficiency II. J Biol Chem 282: 10762-10772.

8. Yoon K, Gaiano N (2005) Notch signaling in the mammalian central nervous system: insights from mouse mutants. Nat Neurosci 8: 709-715.

9. Gray M, Moens CB, Amacher SL, Eisen JS, Beattie CE (2001) Zebrafish deadly seven functions in neurogenesis. Dev Biol 237: 306-323.

10. Appel B, Eisen JS (1998) Regulation of neuronal specification in the zebrafish spinal cord by Delta function. Development 125: 371-380.

11. Appel B, Givan LA, Eisen JS (2001) Delta-Notch signaling and lateral inhibition in zebrafish spinal cord development. BMC Dev Biol 1: 13.

12. Park HC, Appel B (2003) Delta-Notch signaling regulates oligodendrocyte specification. Development 130: 3747-3755.

13. Itoh M, Kim CH, Palardy G, Oda T, Jiang YJ, et al. (2003) Mind bomb is a ubiquitin ligase that is essential for efficient activation of Notch signaling by Delta. Dev Cell 4: 67-82.

14. Salama-Cohen P, Arevalo MA, Grantyn R, Rodriguez-Tebar A (2006) Notch and NGF/p75NTR control dendrite morphology and the balance of excitatory/ inhibitory synaptic input to hippocampal neurones through Neurogenin 3. J Neurochem 97: 1269-1278.

15. Wang Y, Chan SL, Miele L, Yao PJ, Mackes J, et al. (2004) Involvement of Notch signaling in hippocampal synaptic plasticity. Proc Natl Acad Sci U S A 101: 9458-9462.

16. Panzer JA, Gibbs SM, Dosch R, Wagner D, Mullins MC, et al. (2005) Neuromuscular synaptogenesis in wild-type and mutant zebrafish. Dev Biol 285: 340-357.

17. Scheer N, Riedl I, Warren JT, Kuwada JY, Campos-Ortega JA (2002) A quantitative analysis of the kinetics of Gal4 activator and effector gene expression in the zebrafish. Mech Dev 112: 9-14.

18. Willer GB, Lee VM, Gregg RG, Link BA (2005) Analysis of the Zebrafish perplexed mutation reveals tissue-specific roles for de novo pyrimidine synthesis during development. Genetics 170: 1827-1837.

19. Thisse B, Pflumio S, Fürthauer M, Loppin B, Heyer V, et al. (2001) Expression of the zebrafish genome during embryogenesis. ZFIN Direct Data Submission.

20. Thisse B, Thisse C (2005) High Throughput Expression Analysis of ZF-Models Consortium Clones. ZFIN Direct Data Submission.

21. Shin J, Poling J, Park HC, Appel B (2007) Notch signaling regulates neural precursor allocation and binary neuronal fate decisions in zebrafish. Development 134: 1911-1920.

22. Geling A, Steiner H, Willem M, Bally-Cuif L, Haass C (2002) A gammasecretase inhibitor blocks Notch signaling in vivo and causes a severe neurogenic phenotype in zebrafish. EMBO Rep 3: 688-694.

23. Nona SN, Shehab SA, Stafford CA, Cronly-Dillon JR (1989) Glial fibrillary acidic protein (GFAP) from goldfish: its localisation in visual pathway. Glia 2: 189-200.

24. Luhn K, Laskowska A, Pielage J, Klambt C, Ipe U, et al. (2004) Identification and molecular cloning of a functional GDP-fucose transporter in Drosophila melanogaster. Exp Cell Res 301: 242-250.

25. Kochibe N, Furukawa K (1980) Purification and properties of a novel fucosespecific hemagglutinin of Aleuria aurantia. Biochemistry 19: 2841-2846.

26. Lee JS, von der Hardt S, Rusch MA, Stringer SE, Stickney HL, et al. (2004) Axon sorting in the optic tract requires HSPG synthesis by ext2 (dackel) and extl3 (boxer). Neuron 44: 947-960.
27. Thisse B, Thisse C (2004) Fast Release Clones: A High Throughput Expression Analysis. ZFIN Direct Data Submission.

28. Lowe JB (2001) Glycosylation, immunity, and autoimmunity. Cell 104: 809-812.

29. Moloney DJ, Panin VM, Johnston SH, Chen J, Shao L, et al. (2000) Fringe is a glycosyltransferase that modifies Notch. Nature 406: 369-375.

30. Amsterdam A, Nissen RM, Sun Z, Swindell EC, Farrington S, et al. (2004) Identification of 315 genes essential for early zebrafish development. Proc Natl Acad Sci U S A 101: 12792-12797.

31. Golling G, Amsterdam A, Sun Z, Antonelli M, Maldonado E, et al. (2002) Insertional mutagenesis in zebrafish rapidly identifies genes essential for early vertebrate development. Nat Genet 31: 135-140.

32. Hutchinson SA, Eisen JS (2006) Islet1 and Islet2 have equivalent abilities to promote motoneuron formation and to specify motoneuron subtype identity. Development 133: 2137-2147.

33. Cornell RA, Eisen JS (2000) Delta signaling mediates segregation of neural crest and spinal sensory neurons from zebrafish lateral neural plate. Development 127: 2873-2882.

34. Bernardos RL, Lentz SI, Wolfe MS, Raymond PA (2005) Notch-Delta signaling is required for spatial patterning and Muller glia differentiation in the zebrafish retina. Dev Biol 278: 381-395.

35. Bae YK, Shimizu T, Hibi M (2005) Patterning of proneuronal and interproneuronal domains by hairy- and enhancer of split-related genes in zebrafish neuroectoderm. Development 132: 1375-1385.

36. Zecchin E, Filippi A, Biemar F, Tiso N, Pauls S, et al. (2007) Distinct delta and jagged genes control sequential segregation of pancreatic cell types from precursor pools in zebrafish. Dev Biol 301: 192-204.

37. Hegde A, Qiu NC, Qiu X, Ho SH, Tay KQ et al. (2008) Genomewide expression analysis in zebrafish mind bomb alleles with pancreas defects of different severity identifies putative Notch responsive genes. PLoS ONE 3: e1479.

38. Koo BK, Lim HS, Song R, Yoon MJ, Yoon KJ, et al. (2005) Mind bomb 1 is essential for generating functional Notch ligands to activate Notch. Development 132: 3459-3470.

39. Raya A, Kawakami Y, Rodriguez-Esteban C, Buscher D, Koth CM, et al. (2003) Notch activity induces Nodal expression and mediates the establishment of leftright asymmetry in vertebrate embryos. Genes Dev 17: 1213-1218.

40. Harris RJ, Spellman MW (1993) O-linked fucose and other post-translational modifications unique to EGF modules. Glycobiology 3: 219-224.

41. Harris RJ, van Halbeek H, Glushka J, Basa LJ, Ling VT, et al. (1993) Identification and structural analysis of the tetrasaccharide NeuAc alpha $(2 \longrightarrow 6)$ Gal beta $(1->4)$ GlcNAc beta $(1->3)$ Fuc alpha $1->$ O-linked to serine 61 of human factor IX. Biochemistry 32: 6539-6547.

42. Moloney DJ, Haltiwanger RS (1999) The O-linked fucose glycosylation pathway: identification and characterization of a uridine diphosphoglucose: fucose-beta1,3-glucosyltransferase activity from Chinese hamster ovary cells. Glycobiology 9: 679-687.

43. Jaeken J, Matthijs G (2007) Congenital disorders of glycosylation: a rapidly expanding disease family. Annu Rev Genomics Hum Genet 8: 261-278.

44. Sturla L, Rampal R, Haltiwanger RS, Fruscione F, Etzioni A, et al. (2003) Differential terminal fucosylation of N-linked glycans versus protein Ofucosylation in leukocyte adhesion deficiency type II (CDG IIc). J Biol Chem 278: 26727-26733.

45. Choe EA, Liao L, Zhou JY, Cheng D, Duong DM, et al. (2007) Neuronal morphogenesis is regulated by the interplay between cyclin-dependent kinase 5 and the ubiquitin ligase mind bomb 1. J Neurosci 27: 9503-9512.

46. Ohata S, Kinoshita S, Aoki R, Tanaka H, Wada H, et al. (2009) Neuroepithelial cells require fucosylated glycans to guide the migration of vagus motor neuron progenitors in the developing zebrafish hindbrain. Development 136: 1653-1663.

47. Kim J, Irvine KD, Carroll SB (1995) Cell recognition, signal induction, and symmetrical gene activation at the dorsal-ventral boundary of the developing Drosophila wing. Cell 82: 795-802.

48. Rauskolb C, Correia T, Irvine KD (1999) Fringe-dependent separation of dorsal and ventral cells in the Drosophila wing. Nature 401: 476-480.

49. Panin VM, Papayannopoulos V, Wilson R, Irvine KD (1997) Fringe modulates Notch-ligand interactions. Nature 387: 908-912.

50. Nikolaou N, Watanabe-Asaka T, Gerety S, Distel M, Koster RW, et al. (2009) Lunatic fringe promotes the lateral inhibition of neurogenesis. Development 136: 2523-2533.

51. Frydman M, Etzioni A, Eidlitz-Markus T, Avidor I, Varsano I, et al. (1992) Rambam-Hasharon syndrome of psychomotor retardation, short stature, defective neutrophil motility, and Bombay phenotype. Am J Med Genet 44: 297-302. 